\title{
Role of Collagen Derivatives in Osteoarthritis and Cartilage Repair: A Systematic Scoping Review With Evidence Mapping
}

\author{
Germain Honvo (D) · Laetitia Lengelé · Alexia Charles • \\ Jean-Yves Reginster • Olivier Bruyère
}

Received: August 18, 2020 / Accepted: September 23, 2020 / Published online: October 17, 2020

(C) The Author(s) 2020

\begin{abstract}
Introduction: There is currently no diseasemodifying drug for osteoarthritis (OA), and some safety concerns have been identified about the leading traditional drugs. Therefore, research efforts have focused on alternatives such as supplementation with collagen derivatives. The objective of this scoping review is to examine the extent, range, and nature of research, and to summarize and disseminate research findings on the effects of collagen
\end{abstract}

Digital Features To view digital features for this article go to https://doi.org/10.6084/m9.figshare.12987830.

Electronic Supplementary Material The online version of this article (https://doi.org/10.1007/s40744020-00240-5) contains supplementary material, which is available to authorized users.

G. Honvo $(\varangle) \cdot$ L. Lengelé · A. Charles ·

J.-Y. Reginster · O. Bruyère

Division of Public Health, Epidemiology and Health

Economics, University of Liège, Liège, Belgium

e-mail: germain.honvo@uliege.be

G. Honvo · L. Lengelé · A. Charles · J.-Y. Reginster ·

$\mathrm{O}$. Bruyère

World Health Organization (WHO) Collaborating Center for Public Health Aspects of Musculoskeletal Health and Aging, University of Liège, Liège, Belgium

\section{J.-Y. Reginster}

Chair for Biomarkers Research, Biochemistry

Department, College of Science, King Saud

University, Riyadh, Kingdom of Saudi Arabia derivatives in OA and cartilage repair. The purpose is to identify gaps in the current body of evidence in order to further help progress research in this setting.

Methods: The databases Medline, Scopus, CENTRAL, TOXLINE, and CDSR were comprehensively searched from inception to search date. After studies selection against eligibility criteria, following recommended methods, data were charted from the retrieved articles and these were subsequently synthesized. Numerical and graphical descriptive statistical methods were used to show trends in publications and geographical distribution of studies.

Results: The systematic literature search identified a total of 10,834 records. Forty-one published studies were ultimately included in the review, 16 of which were preclinical studies and 25 were clinical studies (including four systematic reviews/meta-analyses). Collagen hydrolysate $(\mathrm{CH})$ and undenatured collagen (UC) were the two types of collagen derivatives studied, with a total of 28 individual studies on $\mathrm{CH}$ and nine on UC. More than a third of studies originated from Asia, and most of them have been published after 2008. Oral forms of collagen derivatives were mainly studied; three in vivo preclinical studies and three clinical trials investigated intra-articularly injected $\mathrm{CH}$. In most of the clinical trials, treatment durations varied between 3 and 6 months, with the shortest being 1.4 months and the longest 11 months. All in vivo preclinical studies and 
clinical trials, regardless of their quality, concluded on beneficial effects of collagen derivatives in OA and cartilage repair, whether used as nutritional supplement or delivered intra-articularly, and whatever the manufacturers of the products, the doses and the outcomes considered in each study.

Conclusions: Although current evidence shows some potential for the use of $\mathrm{CH}$ and $\mathrm{UC}$ as an option for management of patients with $\mathrm{OA}$, there is still room for progress in terms of laboratory and clinical research before any definitive conclusion can be made. Harmonization of outcomes in preclinical studies and longer randomized placebo-controlled trials in larger populations with the use of recommended and validated endpoints are warranted before collagen derivatives can be recommended by large scientific societies.

Keywords: Osteoarthritis; Cartilage repair; Collagen derivatives; Collagen hydrolysate; Undenatured collagen; Scoping review; Evidence mapping

\section{Key Summary Points}

\section{Why carry out this study?}

There are currently no disease-modifying drugs for osteoarthritis (OA) and some safety concerns have been reported regarding the traditional leading anti-OA medications such as paracetamol and non-steroidal anti-inflammatory drugs (NSAIDs).

As an alternative to traditional strategies, there is growing interest in supplementation with collagen derivatives; however, previous literature reviews show that little clinical evidence is available to support their therapeutic benefit for OA patients.
Early clarification of what is currently known about the effects of collagen derivatives in OA and cartilage repair, from preclinical research to real-life studies, appears essential to avoid any unnecessary continuing controversies.

\section{What was learned from the study?}

This scoping review shows that there is relatively little evidence, both from preclinical and clinical research, on the usefulness of collagen derivatives in $\mathrm{OA}$ and cartilage repair; collagen hydrolysate $(\mathrm{CH})$ and undenatured collagen (UC) are the two types of collagen derivatives studied so far, mainly as oral supplements.

All in vivo preclinical studies and clinical trials, regardless of their quality, concluded on beneficial effects of collagen derivatives in $\mathrm{OA}$ and cartilage repair, whether used as nutritional supplement (oral CH or UC) or delivered intraarticularly (intra-articular $\mathrm{CH}$ ). However, three of the four in vitro studies available, all on $\mathrm{CH}$, concluded that $\mathrm{CHs}$ from different sources and of different molecular weights were either ineffective or even detrimental to OA cartilage.

Gaps in the current research include: Limited number of in vitro studies; lack of harmonization of endpoints in preclinical studies; lack of long-term and large-scale randomized, placebo-controlled trials; lack of studies involving patients with hip or hand OA; lack of clinical studies investigating radiological changes in $\mathrm{OA}$ patients after supplementation with collagen derivatives.

\section{DIGITAL FEATURES}

This article is published with digital features, including a summary slide, to facilitate understanding of the article. To view digital features for this article go to https://doi.org/10.6084/ m9.figshare.12987830. 


\section{INTRODUCTION}

Osteoarthritis (OA) is the most common joint disease and a major public health issue of the current century [1]. Due to growing life expectancy, particularly in the developed countries, its global prevalence is rapidly increasing $[1,2]$, making it a disease associated with an extremely high economic burden [2-4]. OA commonly affects the weight-bearing joints and considerably impairs the quality of life of millions of people due to chronic pain and functional limitations $[5,6]$, thus contributing to increase pace of aging, which may ultimately result in premature mortality in the affected people [7].

In the absence of disease-modifying drugs, current strategies for managing OA have relied on a combination of pharmacological and/or non-pharmacological treatments, with the aim of reducing the pain and improving physical function in patients $[8,9]$. Paracetamol and non-steroidal anti-inflammatory drugs (NSAIDs) have been among the most used pharmacological treatments so far [10]. For years, paracetamol has been recommended as the first-line therapy $[11,12]$ despite its low effectiveness to reduce pain in OA [13]. However, recent reports have suggested that this drug is not as safe as it was thought [14] and, consequently, recent guidelines have downgraded the use of paracetamol in OA $[8,9]$. Likewise, safety concerns associated with the use of NSAIDs have limited recommendations for OA patients, particularly for those with comorbidities $[15,16]$. To find alternatives to these drugs, research efforts have focused, since several years now, on medications that would reduce the symptoms of the disease and also be capable of repairing or at least slowing the degradation of articular cartilage. These treatments, known as symptomatic slow action drugs for osteoarthritis (SYSADOAs) are generally considered safe and well tolerated by patients. Currently, glucosamine sulfate (GS) and chondroitin sulfate (CS) are the most used SYSADOAs $[17,18]$, but collagen derivatives are also gaining increasing consideration, mainly as nutritional supplements [19-21].
As $\mathrm{OA}$ is a disease characterized by the progressive destruction of articular cartilage, a major component of which is collagen, it has been postulated that supplementation with collagen hydrolysates may induce the synthesis of cartilage matrix, by stimulating the chondrocytes [19], after intestinal absorption and accumulation in articular cartilage through blood circulation. In fact, experimental studies have demonstrated that peptides from orally administrated collagen hydrolysates accumulated in cartilage tissue a few hours after administration [22-24]. Investigating the efficacy of exogenous administration of collagen derivatives in treating $\mathrm{OA}$ in animal models, recent preclinical studies have reported promising results $[25,26]$, suggesting some potential for cartilage repair in OA patients. These kinds of results have prompted researchers to evaluate the ability of supplementation with collagen derivatives to relieve OA symptoms in humans.

However, previous literature reviews show that little clinical evidence is available to support the therapeutic benefit of collagen derivatives in OA patients [27-29] and furthermore, a systematic literature review with meta-analysis found serious limitations in the methodological quality of these studies [27]. Additionally, almost all of the randomized placebo-controlled trials available on collagen derivatives in $\mathrm{OA}$ are on food ingredients or nutraceuticals, rather than pharmaceutical-grade products [30]. Using data from these clinical trials, the latest metaanalysis found that collagen supplementation had significant positive effect on some (i.e., stiffness) but not the other (i.e., pain and functional limitation) OA symptoms [31]. This lack of strong clinical evidence on the usefulness of collagen derivatives for patients with OA may justify the fact that they are not currently recommended by the leading scientific societies on OA $[8,9,32]$, although largely used by OA patients.

Lessons learned from GS and CS, which have been used for more than two decades as pharmaceutical-grade products and nutraceuticals, should serve for the development and clinical assessment of collagen derivative supplements as adequate alternative options for OA. In fact, 
despite the wide use of these treatments, controversies have surrounded their beneficial effects for patients with OA until currently, due to inconsistent reports from clinical trials $[33,34]$. Ultimately, meta-analyses with subgroup and sensitivity analyses have shown that most of the heterogeneity in results from clinical trials on GS and CS was explained by brand and risk of bias $[35,36]$. Therefore, early clarification of what is currently known about the effects of collagen derivatives on cartilage repair from preclinical research, and on their therapeutic role in human $\mathrm{OA}$ patients appears essential. This might help avoiding unnecessary continuing controversies by helping further designing preclinical research to better clarify the role of collagen derivatives in cartilage repair, in the case of insufficient evidence; as well, it might help better designing future clinical trials, with food-grade or pharmaceutical-grade products manufactured using Good Manufacturing Practice.

Thus, the objective of this scoping review is to examine the extent, range, and nature of research, and to summarize and disseminate research findings on the effects of collagen derivatives on cartilage and specifically on articular cartilage, as well as on the effects of these compounds as a symptomatic and/or chondroprotective treatment in $\mathrm{OA}$ patients. Our purpose is to identify research gaps in the available evidence and to progress research on this potentially promising therapeutic or adjuvant option for OA patients.

\section{METHODS}

This scoping review was conducted according to the guidance developed by the Joanna Briggs Institute (JBI), which was first published in 2015 [37] and updated in 2017. We also followed recommendations by Arksey and O'Malley [38] and those by Levac, Colquhoun and $\mathrm{O}^{\prime}$ Brien [39]. The Covidence online software was used to manage the entire review process, including screening of records based on title and abstracts, and full texts screening. The findings of this research were reported according to the Preferred Reporting Items for Systematic reviews and Meta-Analyses extension for Scoping Reviews (PRISMA-ScR) guidelines [40]. This article is based on previously conducted studies and does not contain any studies with human participants or animals performed by any of the authors.

\section{Protocol and Registration}

The protocol of this scoping review was not registered in PROSPERO, as scoping reviews do not fall into the scope covered by this database. However, the protocol, dated February 29, 2020, is available from the authors to anyone who would like to have access to it.

\section{Eligibility Criteria}

\section{Inclusion Criteria}

Pre-clinical studies (in vitro and in vivo laboratory animal studies) on the effects of collagen derivatives on any type of cartilage, clinical studies (interventional studies), epidemiological non-interventional studies (observational studies), and post-marketing surveillance studies assessing the symptomatic and/or chondroprotective effects of collagen derivatives in patients with osteoarthritis were included in this scoping study, regardless of the study design. Studies on combination products (i.e., collagen derivatives combined with anti-OA medication) were also included, as were studies simultaneously involving patients with $\mathrm{OA}$ and patients with diseases other than OA. Finally, evidence synthesis studies, such as systematic literature reviews and meta-analyses, were considered for inclusion.

\section{Exclusion Criteria}

Clinical trials, epidemiological non-interventional studies, and post-marketing surveillance studies in veterinary medicine were excluded, as were studies on collagen as biomarker of OA or other diseases. We also excluded studies using collagen as a carrier for other therapies (e.g., stem cells or chondrocytes for transplantation), as well as studies using collagen as a scaffold for cartilage repair. Simple literature reviews (nonsystematic reviews), letters, comments, and 
editorials were not considered for inclusion in this scoping study. No date limitation was applied to the search, however articles in languages other than English or French were excluded; this language restriction has been chosen because of time constraints for translating articles from other languages, as no member of the research team has competences in languages such as Chinese, Japanese, or Russian, etc.

\section{Information Sources and Search Strategies}

We comprehensively searched the following literature databases, with search strategies adapted to each database: Medline (Ovid), Scopus, Cochrane Central Register of Controlled Trials (Ovid CENTRAL), TOXLINE (ProQuest), and Cochrane Database of Systematic Reviews (Ovid CDSR). Each database was searched from inception to February 28, 2020.

The search strategies were constructed using combinations of words describing the population/disease of interest ("osteoarthritis" or "cartilage"), the studied concept ("collagen derivatives"), and the context ("treatment" [of osteoarthritis]; [cartilage] "repair" or "regeneration"). Depending on the database, combinations of free vocabulary words and/or controlled terms were used. The detailed search strategies for all databases are shown in the appendix to this article (Electronic supplementary file 1), except that of the Cochrane Database of Systematic Reviews, as the search of that database returned no result.

The reference list of systematic reviews that were included were hand-searched for additional studies that would not have been found through databases search.

Electronic clinical trial registries such as ClinicalTrials.gov, the World Health Organisation International Clinical Trial Registry platform (WHO ICTRP), the International Standard Randomised Controlled Trial Number (ISRCTN) registry and the European Union Clinical Trials Register, as well as the Clinical Trials RegistryIndia (CTRI) and the Chinese Clinical Trial Registry (ChiCTR) were also searched for potential ongoing clinical trials on collagen derivatives in $\mathrm{OA}$.

\section{Selection of Sources of Evidence}

Two reviewers (GH and LL) independently screened the titles and abstracts of the records to identify all potentially relevant studies. Once a selection decision was made by the two reviewers on all records, a consensus meeting was set up to solve disagreements. If the relevance of a study was unclear or if disagreements persisted based on the information in the abstract, the study was included for more information in the full text. After this step, the full texts of the selected studies were searched, and a new selection process was undertaken independently by the two reviewers (GH and LL), based on full article screening against the eligibility criteria. Studies that were selected by the two review authors were automatically included in the review. A consensus meeting was organized to make a final decision regarding studies for which the review authors took divergent selection decisions.

\section{Data Charting Process and Data Items}

Three reviewers (LL, AC, and GH) were involved in the data charting process. This consisted in charting key items of information relevant to the objective of the scoping review, from the primary research articles. One of the reviewers (LL) charted the data from preclinical studies, and the second reviewer (AC), from clinical studies. Then, the data charted from each category of research papers (preclinical and clinical) by the first two reviewers were cross-checked by the third reviewer $(\mathrm{GH})$ while making the synthesis of studies, to detect and correct any mistake in the extracted data. All the synthetized data were checked again by the three review authors before the submission of the manuscript for publication.

The data charting process was conducted using pre-designed forms. As the nature of key information may vary according to the types of studies [39], we designed specific data charting forms for preclinical and clinical studies. These 
forms were pre-tested by the team before engaging in the formal data charting process.

We extracted, from each study, general information to identify the study, and more specific information on the study population, information on the type and characteristics of collagen derivative studied, information on the methodology of the study, and finally, the main result(s) and conclusion(s) of the study. More specifically, the following extraction fields were considered: Authors of the study (first author only), year of publication, name of the journal, country of origin of the study, objective/aim/ purpose of the study, study population, sample size, type and source of collagen, administration route, outcome(s) of interest, study design, brief description of the methodology of the study, duration of the study, outcomes measurement tools, key findings of the study, limitation(s) of the study according to the authors, conclusion(s) of authors, aeras of uncertainty and future direction of research (perspectives), as suggested by the authors.

In the case of missing important information in the published articles, the authors were contacted by e-mail to get the missing data. Because of time constraints, we defined a deadline of a week, after having sent the e-mail, for receiving missing data from authors, after which the missing data were considered as "unavailable data".

\section{Critical Appraisal of Individual Sources of Evidence}

As explained by Arksey and O'Malley [38], and further outlined in the guideline developed by the Joanna Briggs Institute for conducting scoping reviews [37], a formal assessment of the methodological quality or risk of bias of the included studies was not performed. Indeed, this is out of the scope of scoping reviews.

\section{Synthesis of Results}

We created a PRISMA flow chart of study selection to report the studies selection process with the number of included and excluded studies at each step of the selection; specific numbers with reasons of full texts exclusions were explicitly reported in the flow chart. The key information from each article, as described in the data items section, were reported in tables summarizing the main characteristics and findings of the included studies. Preclinical studies and clinical studies were described separately to ease understanding, given that these two kinds of research papers do not necessary report the same type of key information.

We used a narrative approach to report information from the included studies, in addition to the summary provided in tables, with no attempt to aggregate findings from various studies, as this is not the aim of a scoping review. We presented numerical and/or graphical syntheses of the extent, nature, and distribution of the different types of studies retrieved from our literature search, using methods of descriptive statistics. Where appropriate, graphical representations were preferred to visualize various aspects related to the research activity on the effects of collagen derivatives on articular cartilage and OA, using the STATA and Microsoft Excel software. We used choropleth world maps (drawn using Microsoft Excel 2019) to show the geographical distribution of studies, trend graphs to visualize the trends in publications on collagen derivatives over time, and a bar diagram to depict the relative frequency of each specific type of study.

\section{RESULTS}

\section{Literature Search}

Our electronic databases search yielded 10,825 records, and nine additional records were identified through manual search and clinical trials registries search. After duplicates were removed, 9405 records were screened based on title and abstracts. This primary screening led to 99 full texts that were assessed against the eligibility criteria. Ultimately, 48 studies were included in the scoping review. All the detailed review process and reasons for full-text exclusions with numbers of excluded full texts are shown in Fig. 1. 

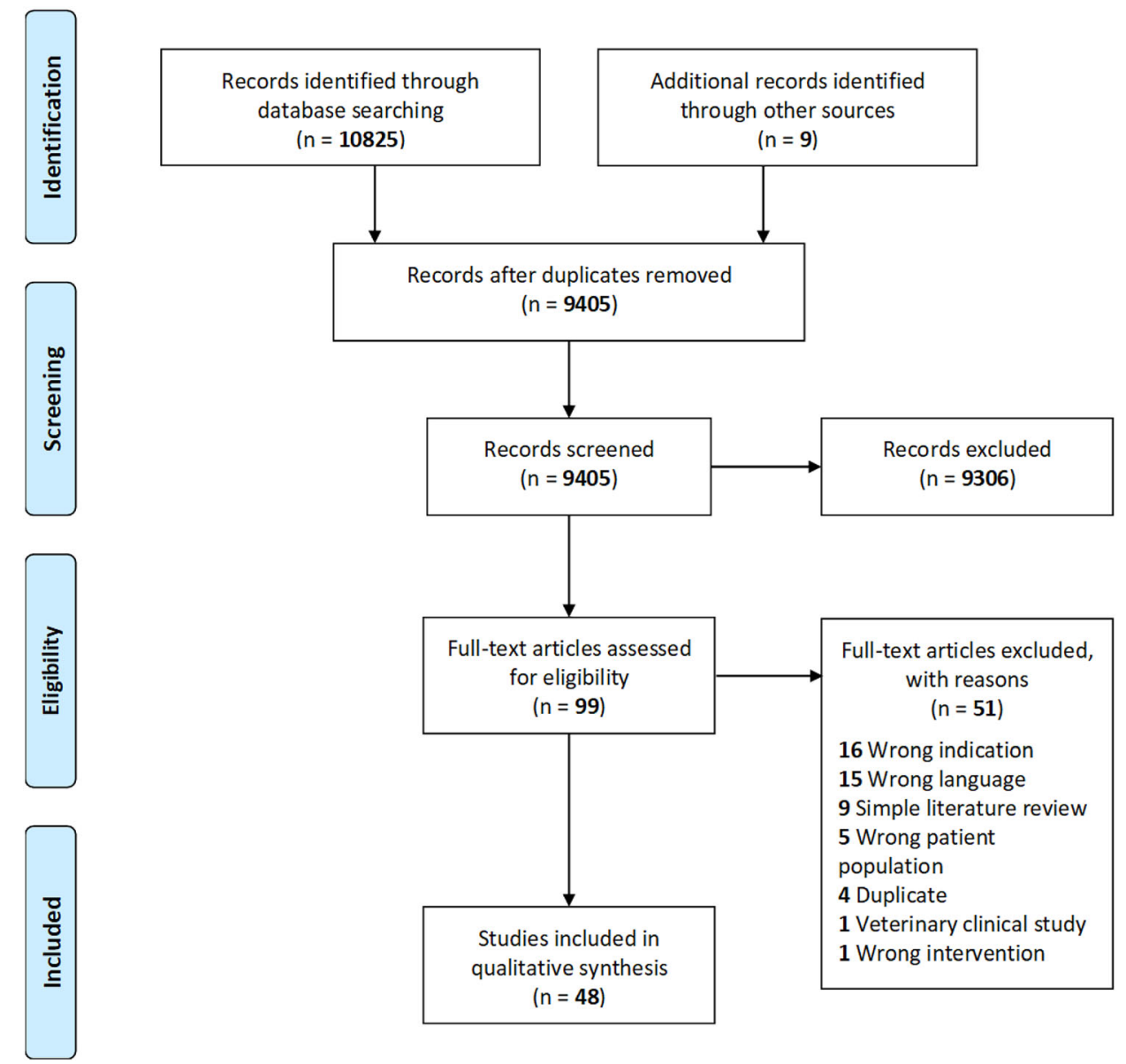

Fig. 1 PRISMA flow diagram of the scoping review

\section{Description of Evidence on Collagen Derivatives in OA and Cartilage Repair}

\section{Types of Studies}

A total of 41 studies have been published on collagen derivatives in $\mathrm{OA}$ and cartilage repair so far. Sixteen of these studies were preclinical in vitro and in vivo studies $[25,26,41-54]$ and 25 were clinical studies (including clinical trials, observational studies, and systematic reviews/ meta-analyses) $[27,28,31,55-76]$. Figure 2 depicts the distribution of the studies by specific study type. The most published studies are clinical trials (15 publications), followed by in vivo preclinical studies (12 publications). There are currently four published in vitro studies on effects of collagen derivatives on cartilage, six observational studies, and four systematic reviews/meta-analyses that have assessed the symptomatic effects of collagen derivatives in OA patients.

We identified seven studies registered in clinical trial registries, of which five were reported as completed; however, we were unable to find any article reporting the results of these studies. For one of the studies identified in clinical trial registries, the recruitment status was reported as unknown; the other one was reported as recruiting (see Table S1 in the electronic supplementary material for details).

\section{Trends in Publication of Evidence}

To visualize the trends in publication of studies on collagen derivatives in $\mathrm{OA}$ and cartilage repair over time, we plotted the number of studies published by year against the years of publications. Figure 3 shows the trends in total publication of studies (overall preclinical and 


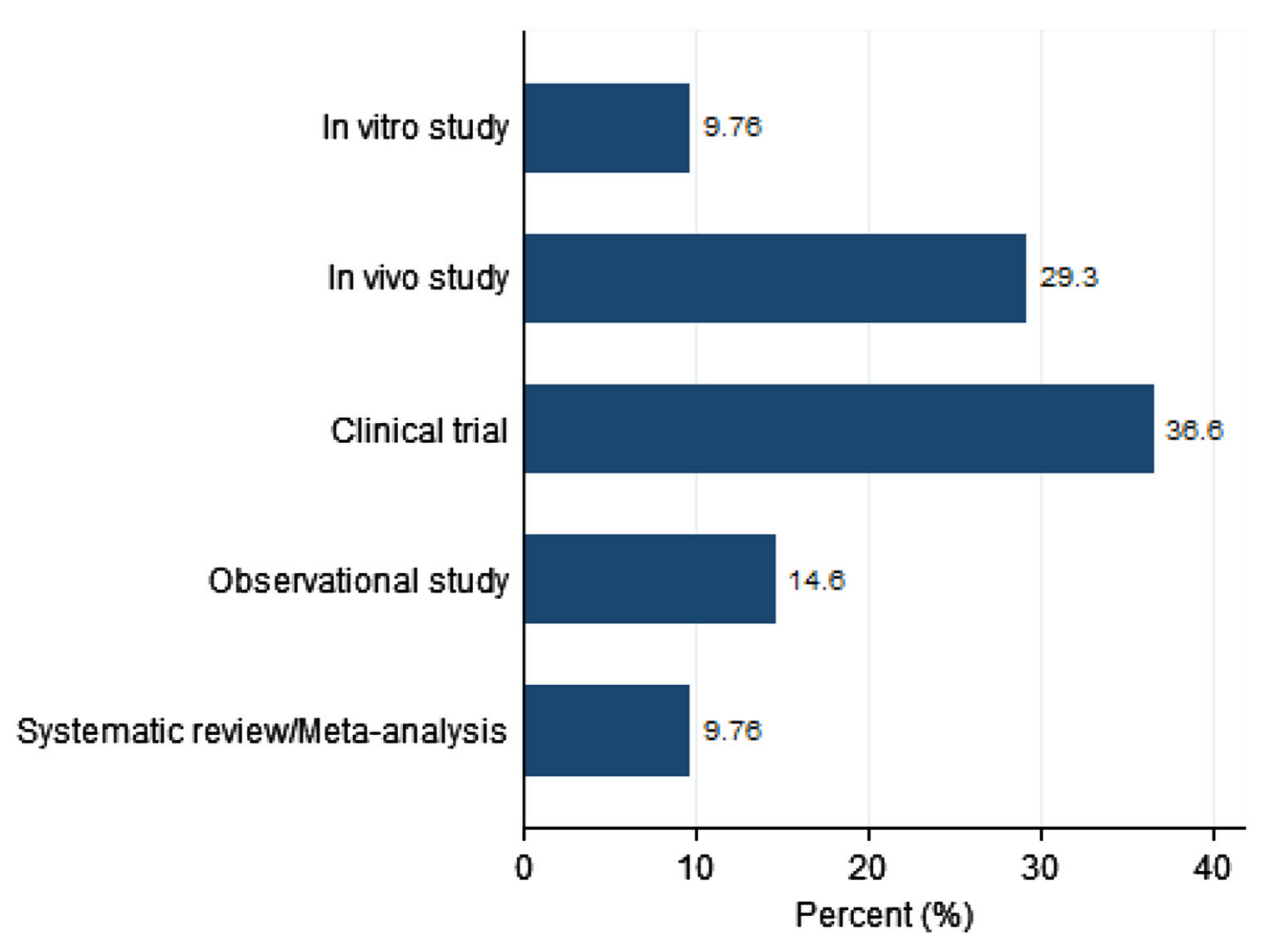

Fig. 2 Distribution of published studies on collagen derivatives for osteoarthritis and cartilage repair, by study type

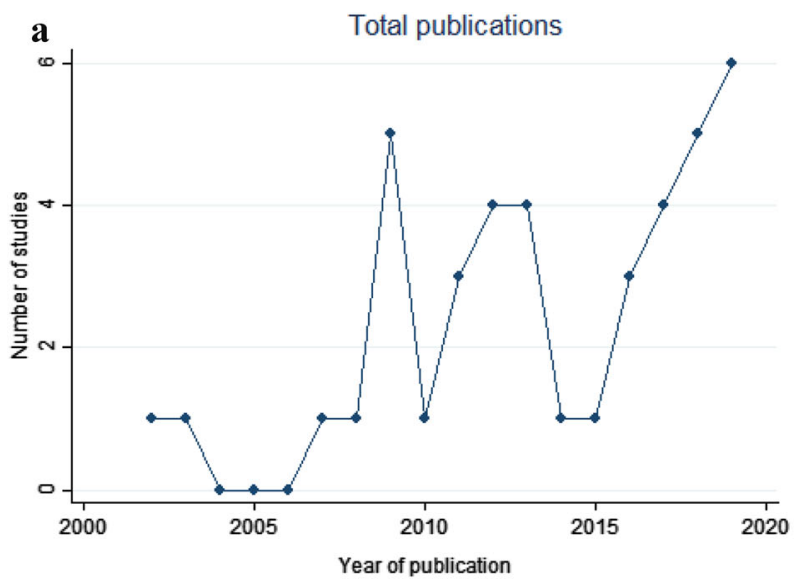

Fig. 3 Trends in publication of studies on collagen derivatives for osteoarthritis and cartilage repair [the top figure is about total publications (a) and the second

clinical studies), as well as the comparative trends in publication of preclinical and clinical studies (including systematic reviews/metaanalyses), beginning from the year of the first published study (2002) to the year of the last published study (2019), at the date of our literature search (February 28, 2020).

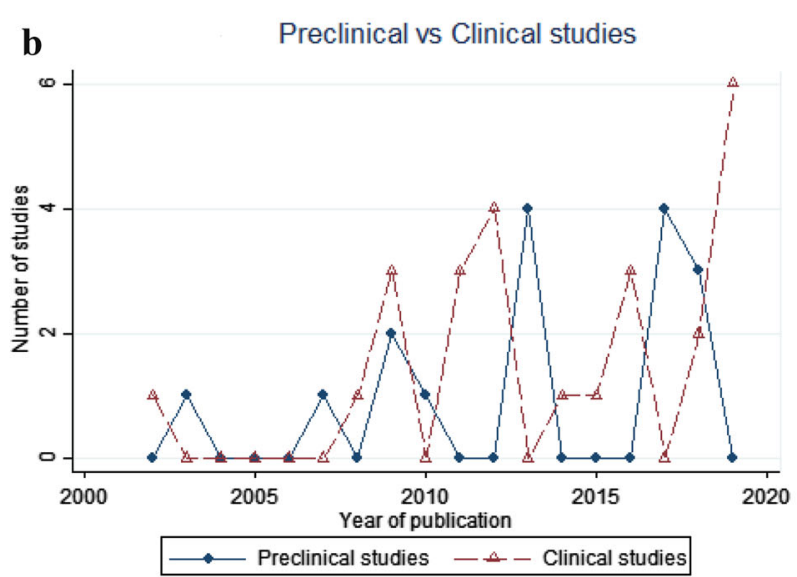

figure compares trends in publication of preclinical and clinical studies (b), including systematic reviews and metaanalyses)

As shown by that figure, very few studies were published before 2009 (four studies in total), with an increase in publications from 2009. It is remarkable that at least one study was published by year since 2009, with picks in publications in 2009 (five studies) and 2019 (six studies). After variations in numbers of total 
publications by year, a slight tendency to a progressive increase is observed since 2016. The comparison of the trends in publications of preclinical studies to that of clinical studies shows important fluctuations in annual publications of each of the two types of research.

\section{Geographical Distribution of Studies}

The geographical distribution of publications is shown on choropleth world maps (Fig. 4), with numbers of studies by country proportionate to color intensity: the darker the color, the higher the value. For all preclinical studies, the study country is the country of the research center in which the study was conducted. For clinical studies, the study country is the country of the leading author of the publication, which sometimes is not the country in which the study was conducted (i.e., patient recruitment country for clinical trials).

As can be seen in Fig. 4, most of the studies originate from Asia. Very few preclinical studies have been conducted in European countries (three studies) and in USA (two studies). In fact, most of these studies originated from Japan (six studies) and China (three studies). Clinical studies are rather better distributed across the world, with eight studies from European countries (including two systematic reviews/metaanalyses), two from Turkey, three from the USA, and seven from Asian countries (four studies from India). An important note is that none of

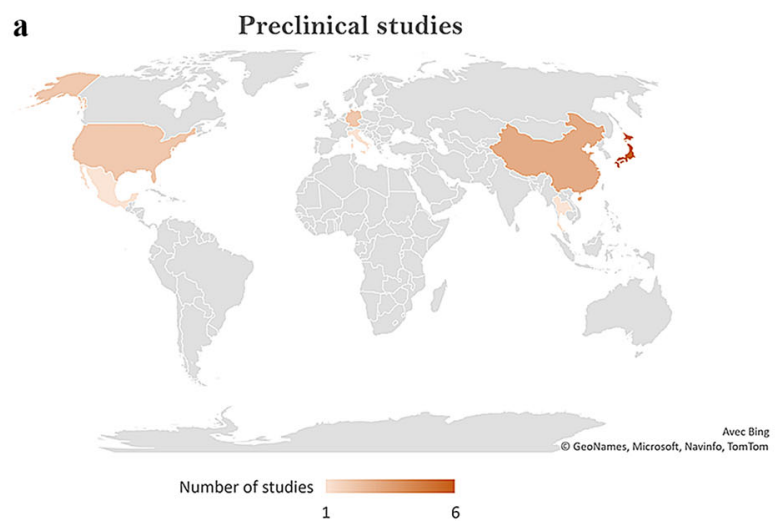

the identified clinical studies originated from Japan, which concentrates the highest number of preclinical studies.

\section{Synthesis of Evidence on Collagen Derivatives}

\section{Preclinical Studies}

Four in vitro studies have evaluated the effects of collagen from different sources and of different molecular weights on articular cartilage explants (Table 1). All these studies investigated collagen hydrolysate $(\mathrm{CH})$. Cartilage metabolism and degradation outcomes, as well as levels of various inflammatory mediators, were measured. Three of these studies, assessing different preparations of $\mathrm{CH}$, from different sources and with different molecular weights, found that $\mathrm{CH}$ was either ineffective or detrimental to articular cartilage in pathological condition $[41,43,44]$, suggesting that collagen preparations from various sources might have different peptide composition and differ significantly in their effects on articular cartilage (see conclusions in Table 1). All of these studies advocated for further research in this setting. None of them was supported by pharmaceutical companies.

Our systematic literature search identified 12 in vivo studies assessing the effects of collagen derivatives in different animal models (Table 1). Only two of these studies [25, 47] evaluated

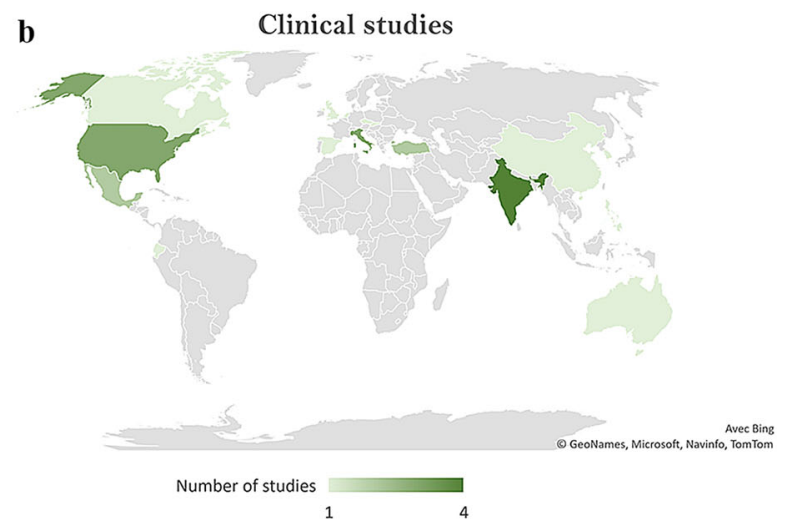

Fig. 4 Geographical distribution of studies on collagen derivatives for osteoarthritis and cartilage repair; a preclinical studies and $\mathbf{b}$ clinical studies 


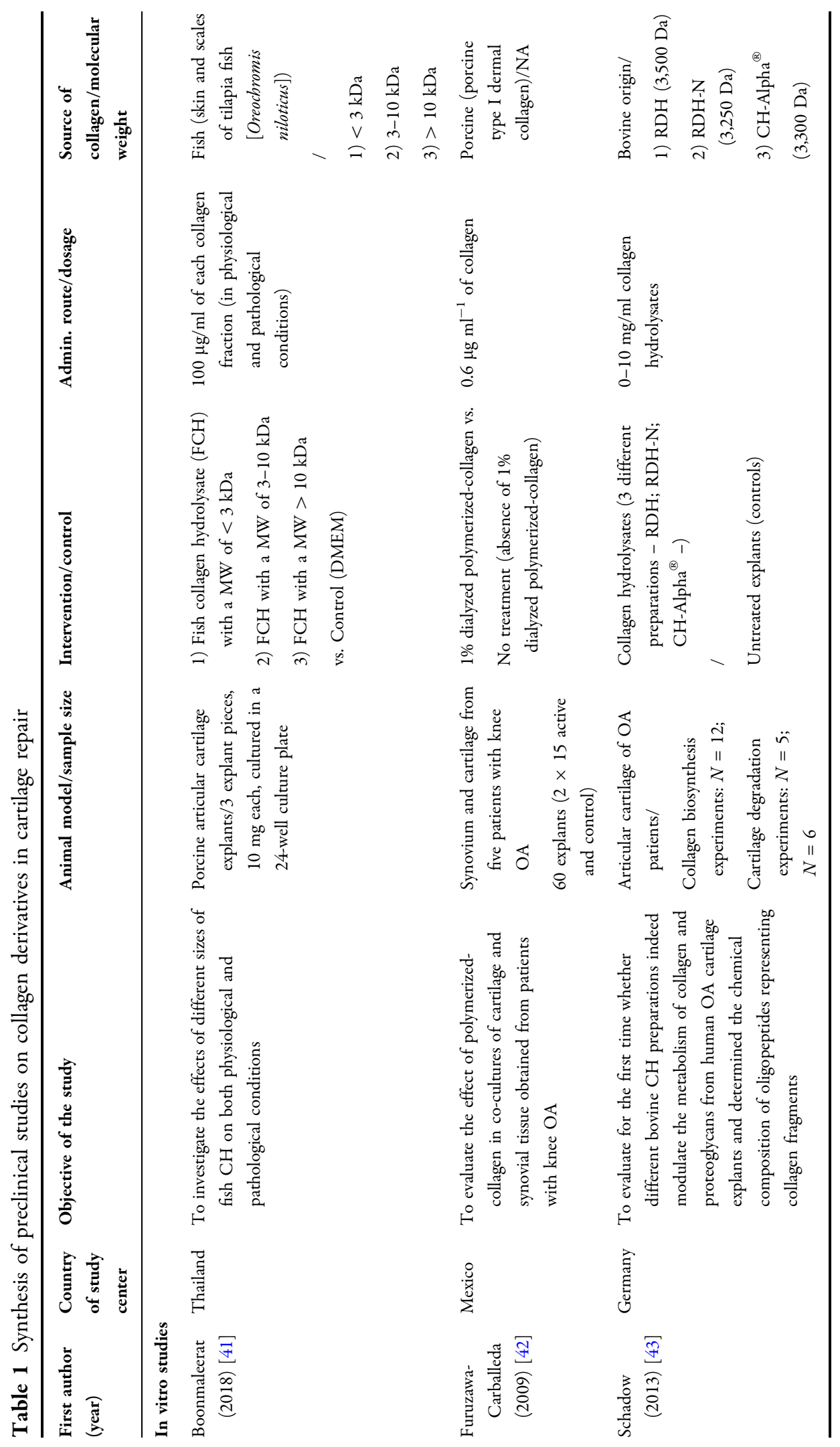




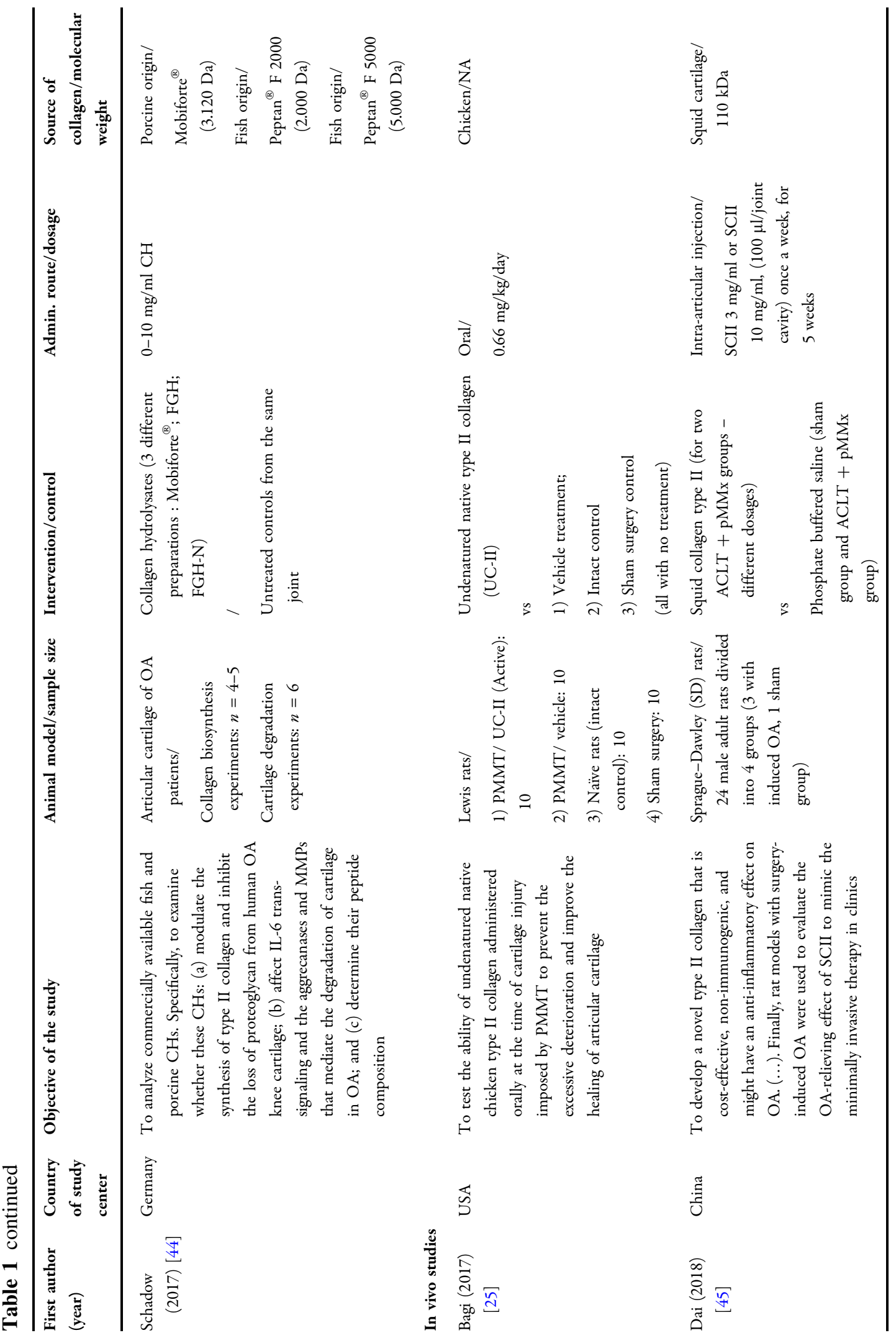




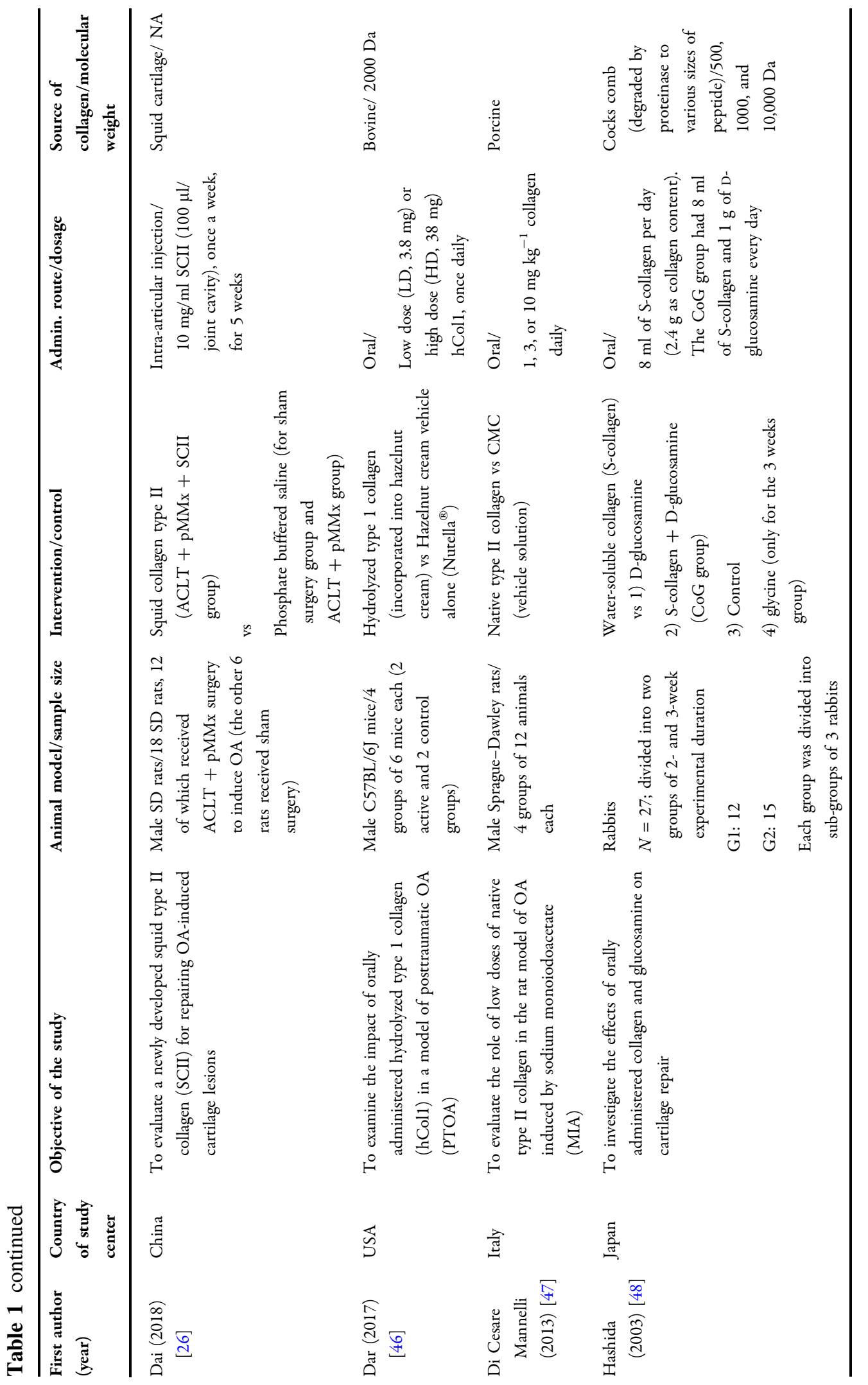




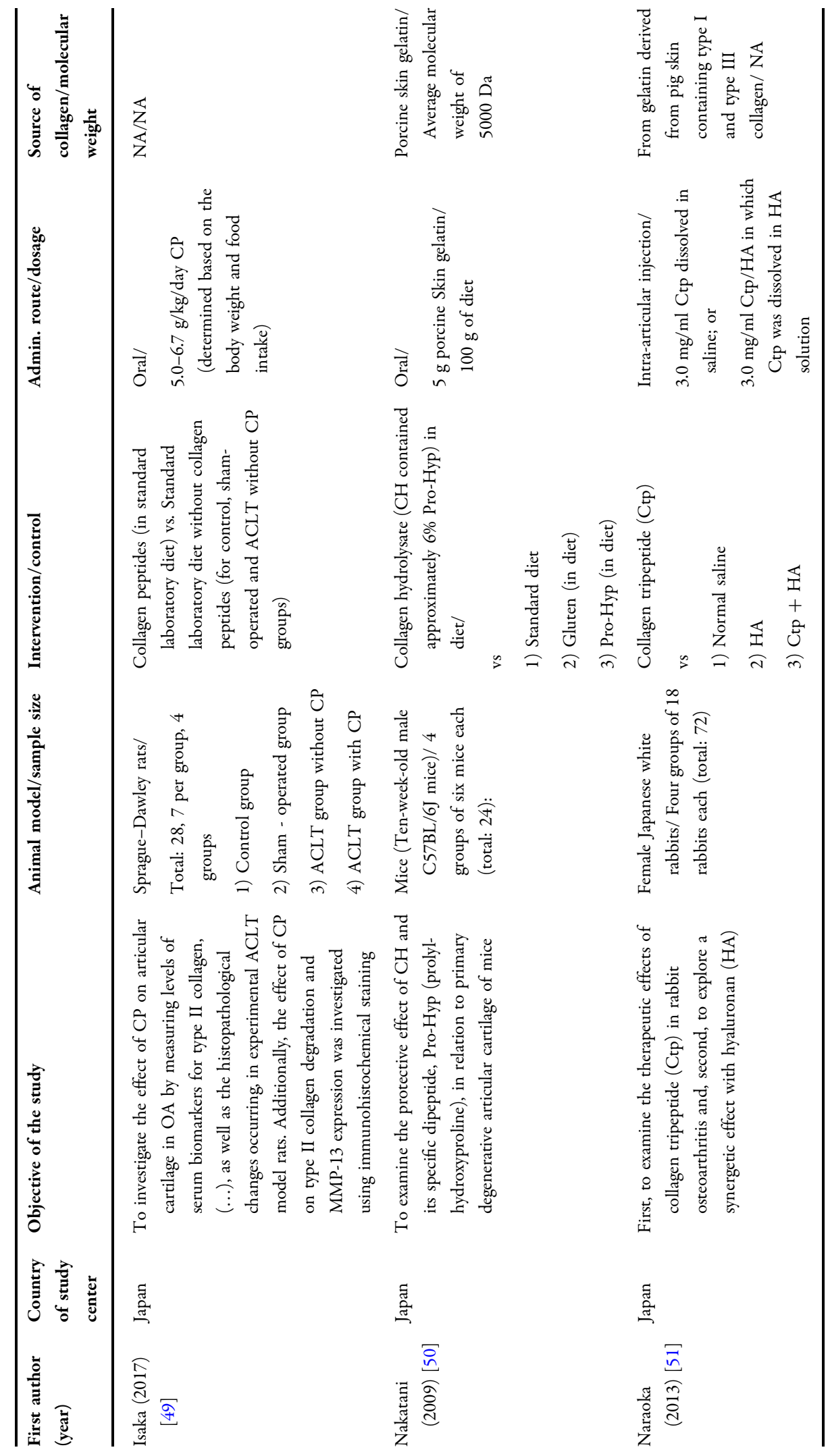




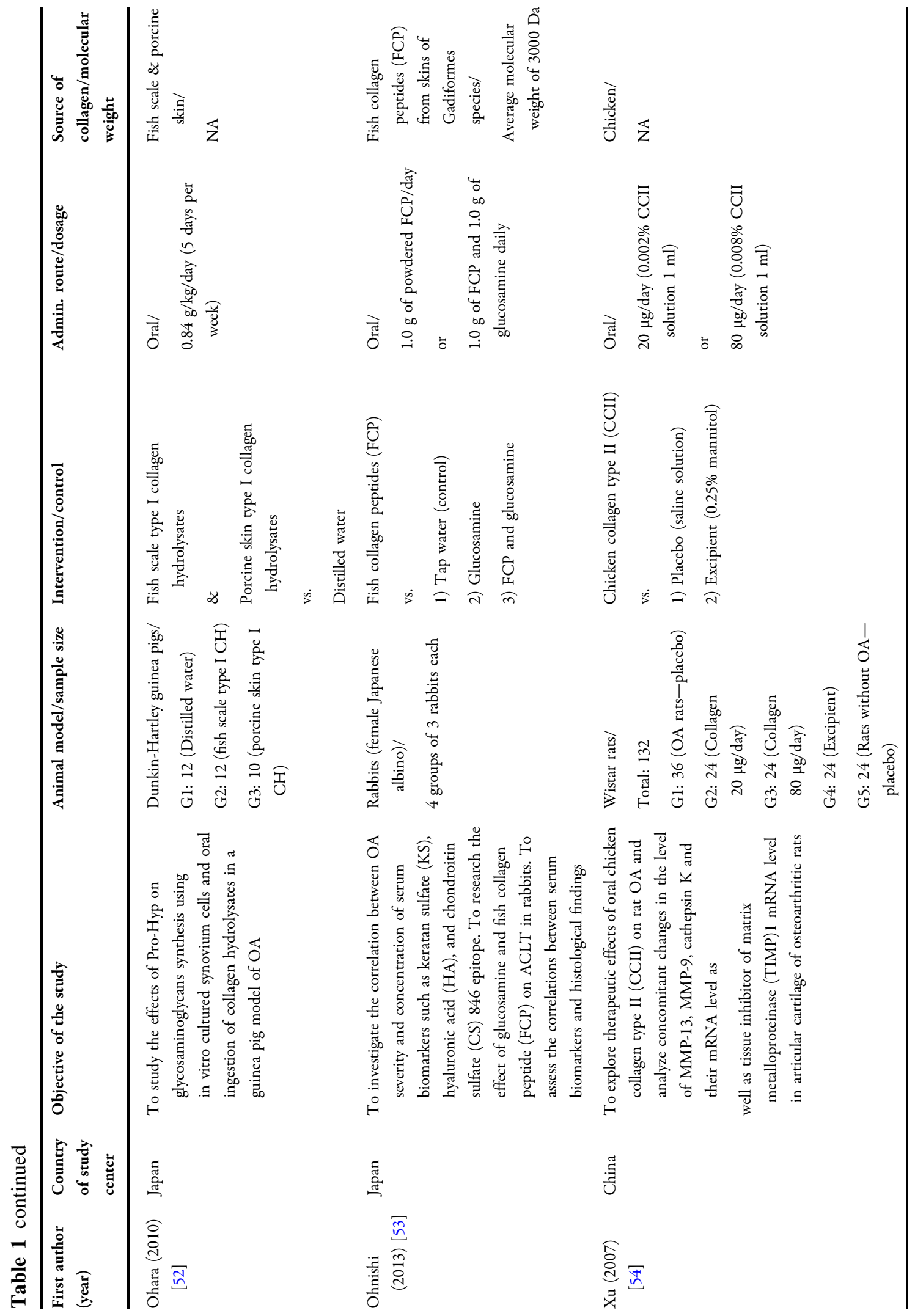




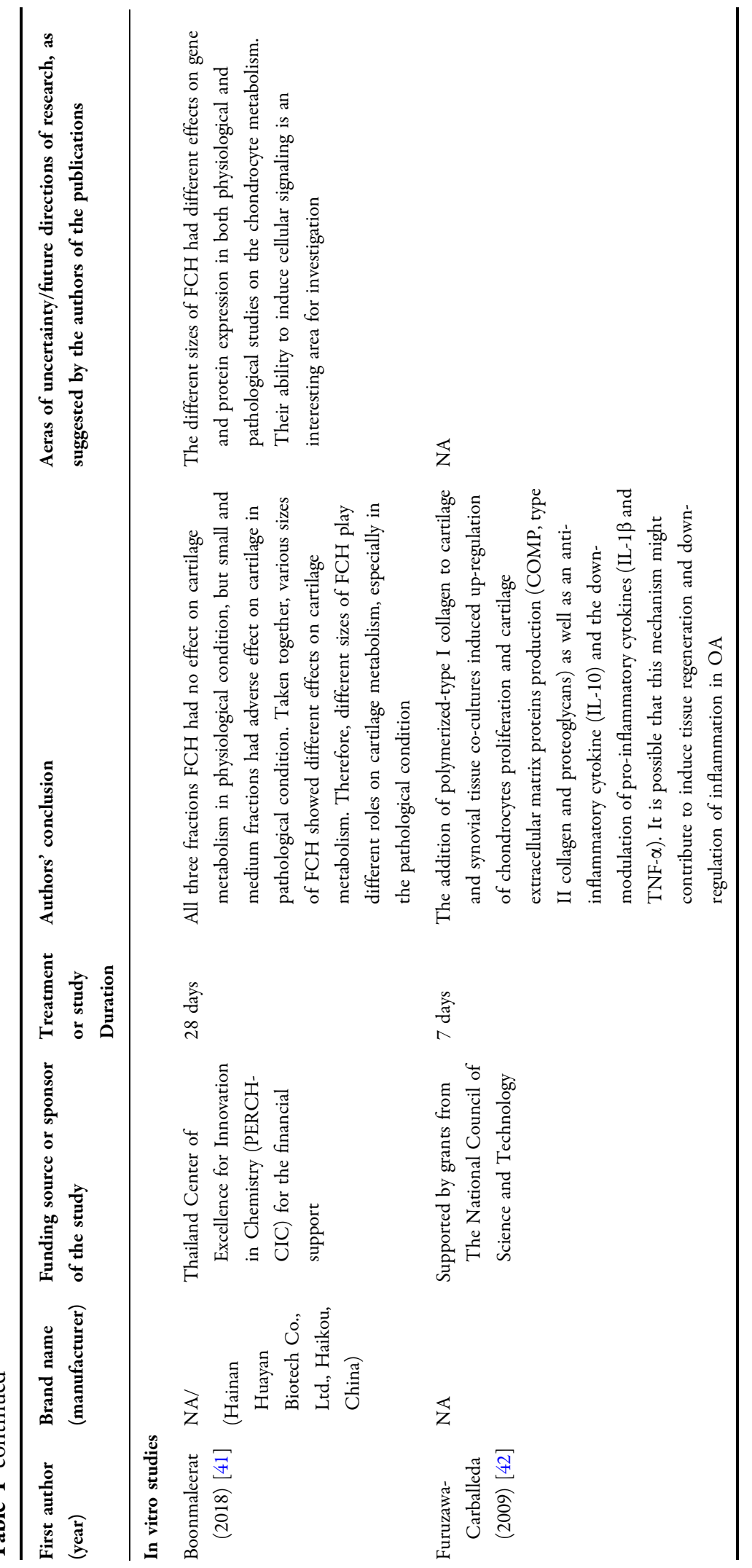




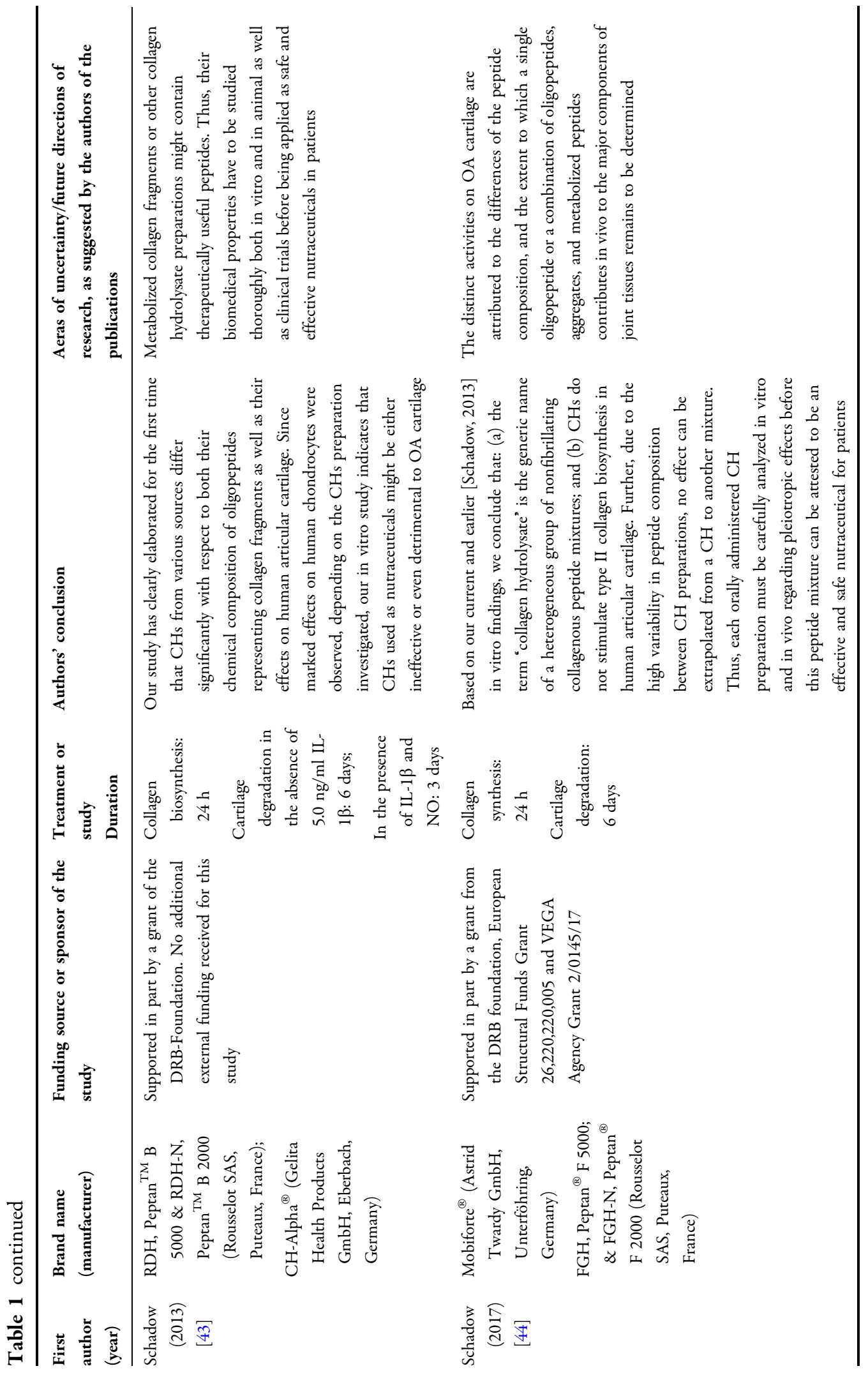




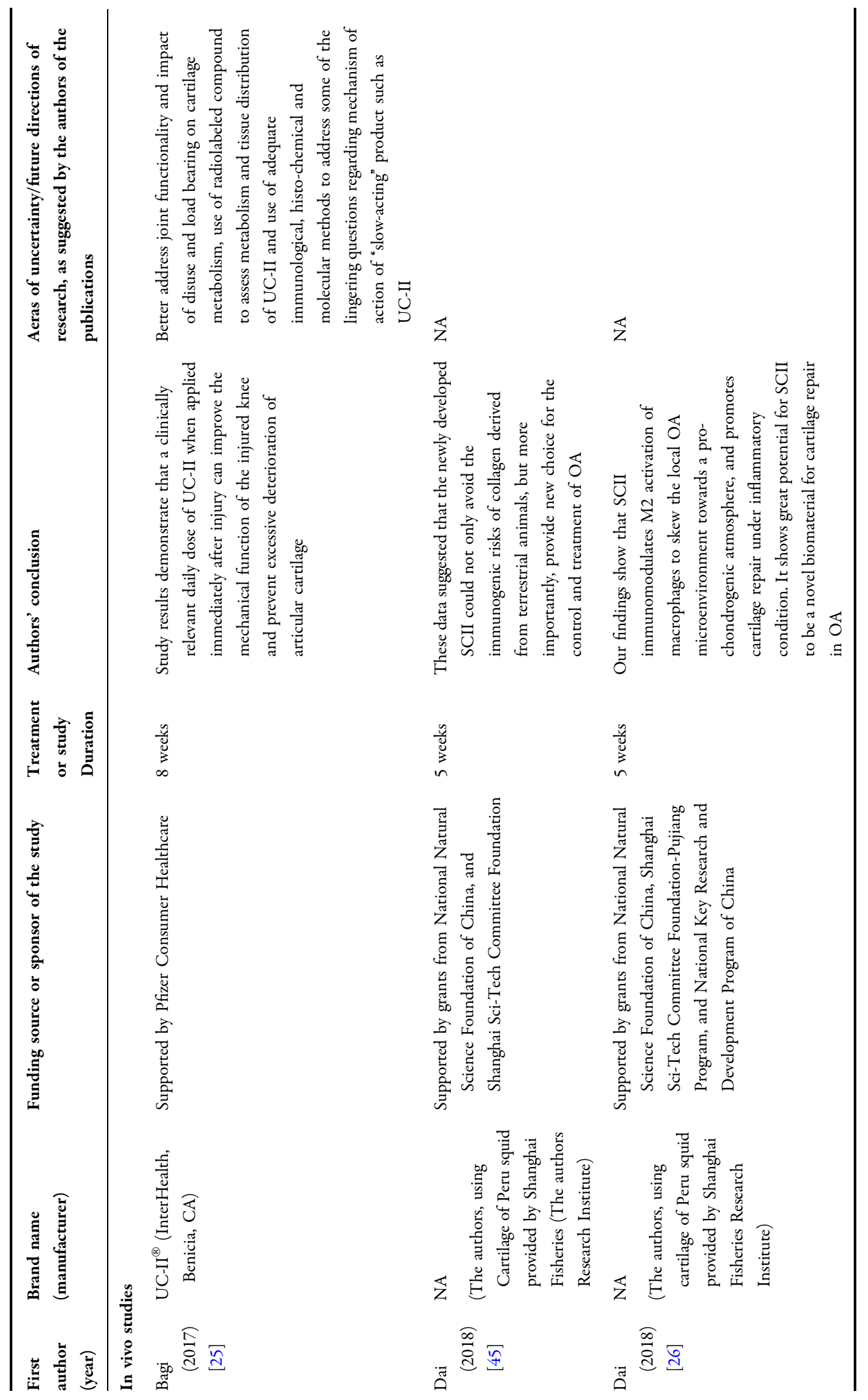




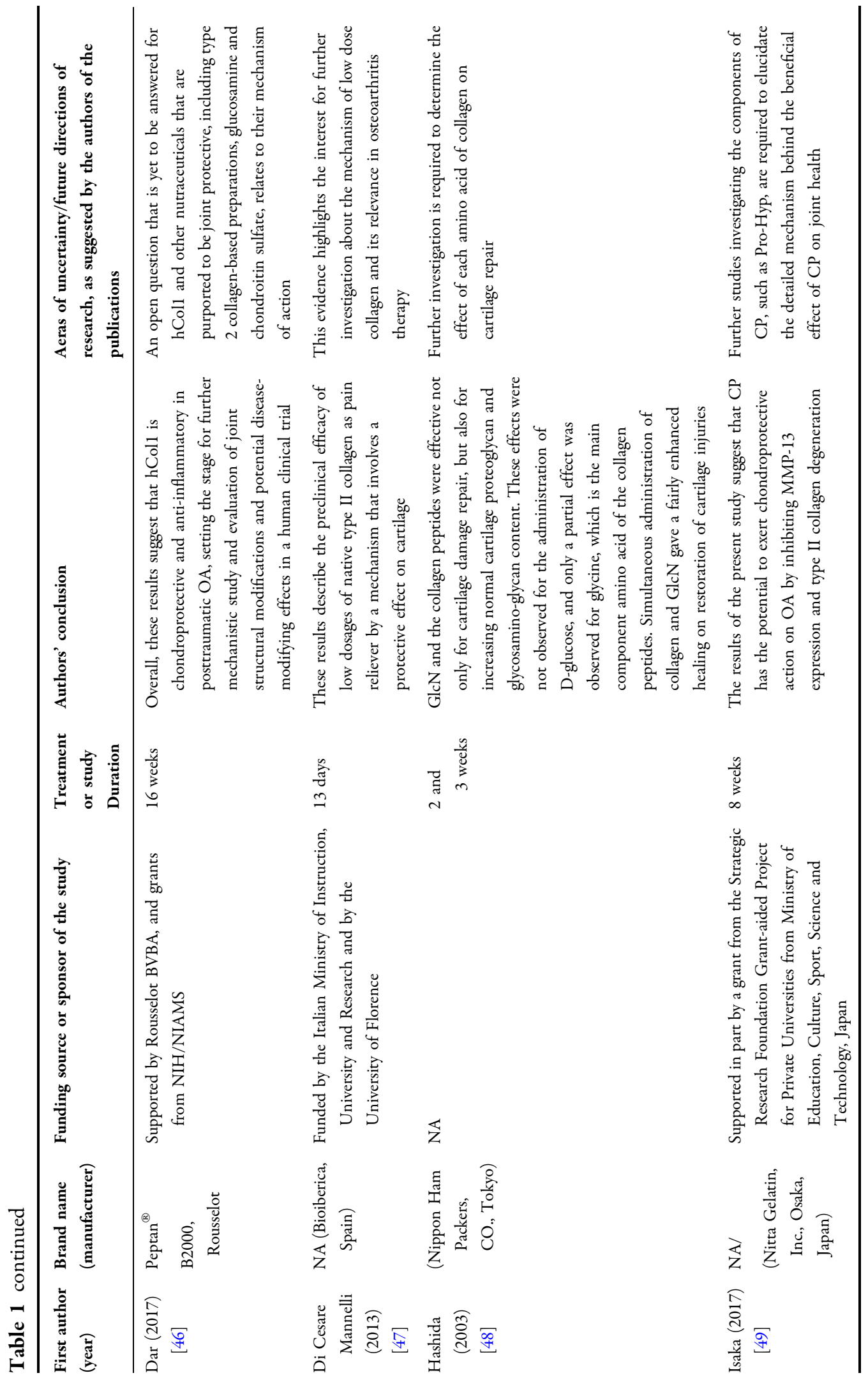




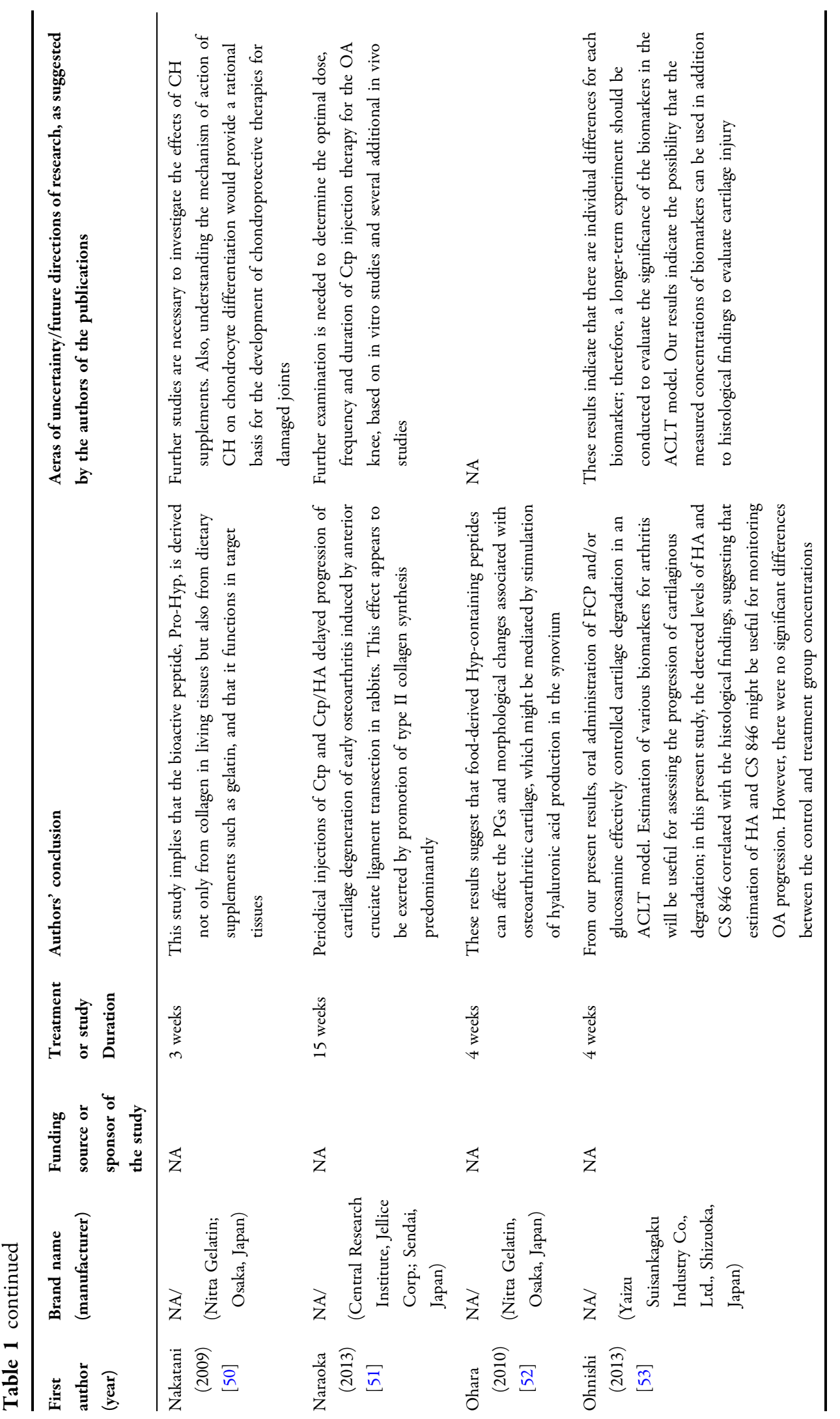




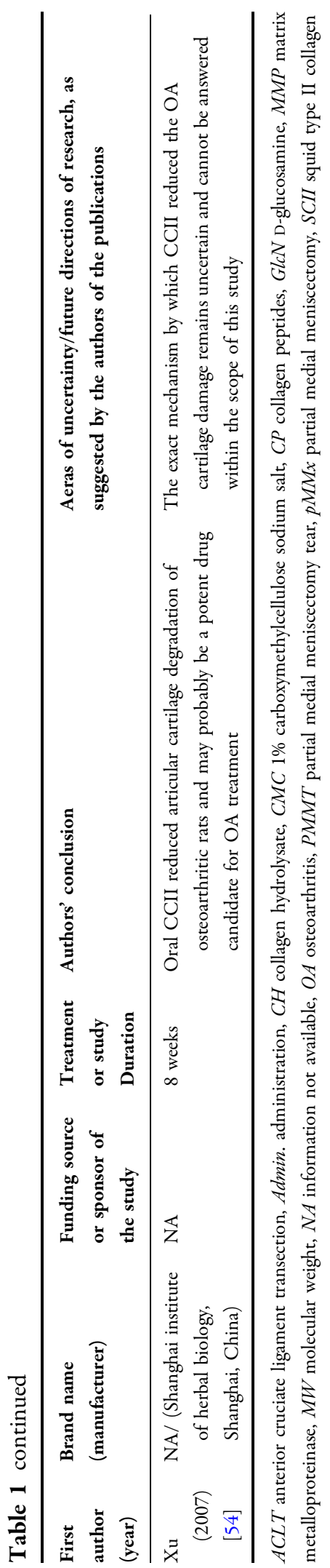

undenatured collagen (UC), also known as native collagen. The other ten studies investigated collagen hydrolysate $(\mathrm{CH})$; two of them used atelocollagen, administered by intra-articular injection $[26,45]$. The two studies on UC used different rat models of OA, different dosages of orally administered collagen (see Table 1), and measured different outcomes [25, 47]. In Bagi et al. [25], the endpoints included the weight-bearing capacity of front and hind legs, serum biomarkers of bone and cartilage metabolism, analyses of subchondral and cancellous bone at the tibial epiphysis and metaphysis, cartilage pathology at the medial tibial plateau using histological methods, and the presence of possible fractures or other bone abnormalities. In the study by Di Cesare Mannelli et al. [47], the following outcomes were measured: The decrease of articular pain, postural unbalance measured as hind-limb weightbearing alterations, motor activity, cartilage degradation measured by the decrease of plasma and urine levels of cross-linked C-telopeptide of type II collagen (CTX-II). Although these two studies did not consider the same outcomes, they all concluded that UC have protective effect on articular cartilage. Likewise, the studies on $\mathrm{CH}$, investigating collagen from various sources and at different dosages, all concluded that this compound has a chondroprotective effect.

\section{Clinical Studies}

All the retrieved clinical trials and observational studies were designed to assess the efficacy and/ or safety of collagen derivatives in OA patients. The main characteristics and findings of these studies are summarized in Table 2 . Almost all of these studies included patients with knee OA; only one RCT [67] and two observational studies $[74,75]$ included patients with hand, hip, or knee OA. The clinical trials investigated UC and $\mathrm{CH}$, and there were more RCTs on $\mathrm{CH}$ than on UC.

Five clinical trials investigated UC, among which only two studies were RCTs against placebo, namely the studies by Stančík et al. [68] and Lugo et al. [64]. The first study investigated undenatured type I collagen (COL-I), and the second was on undenatured type II collagen 


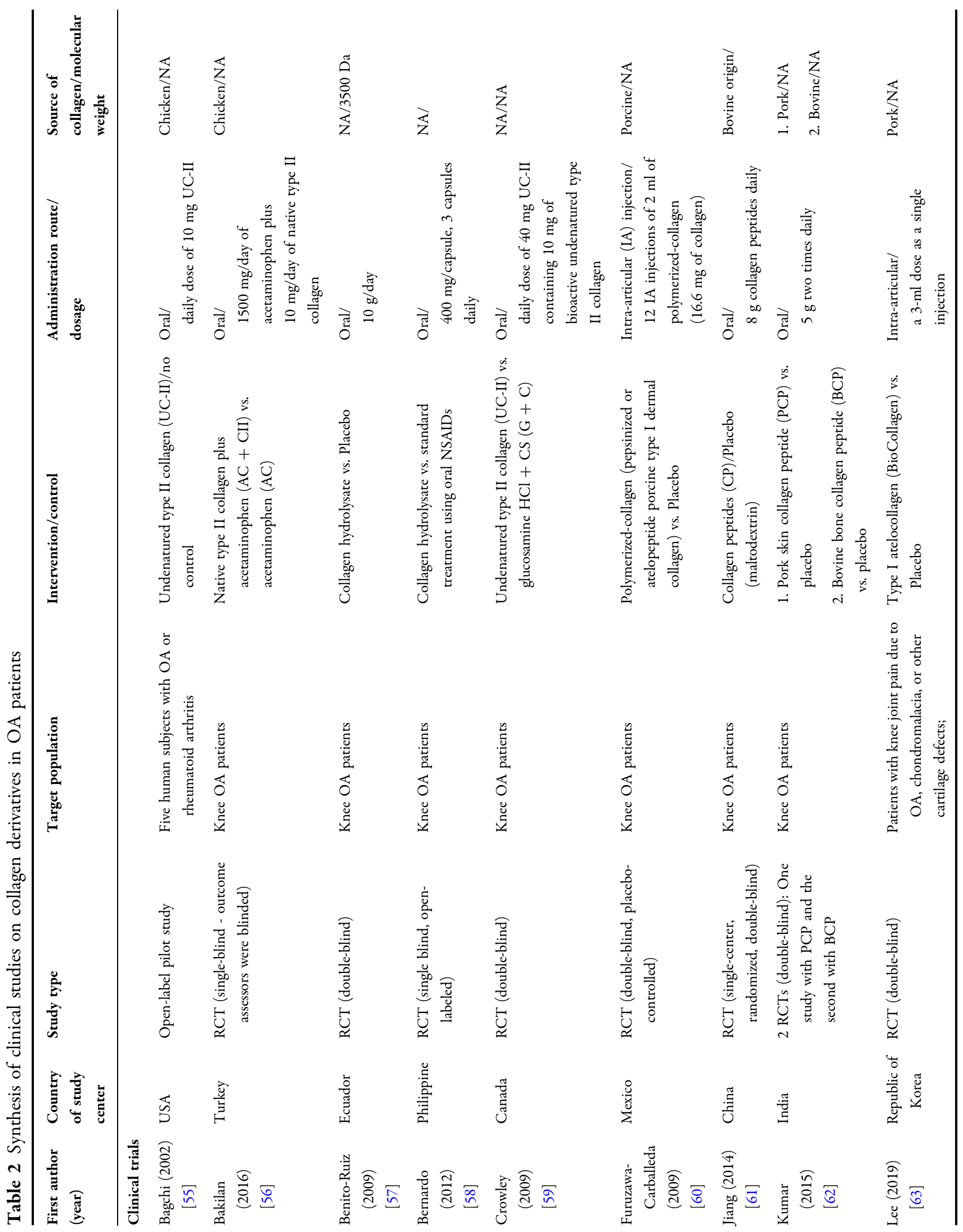




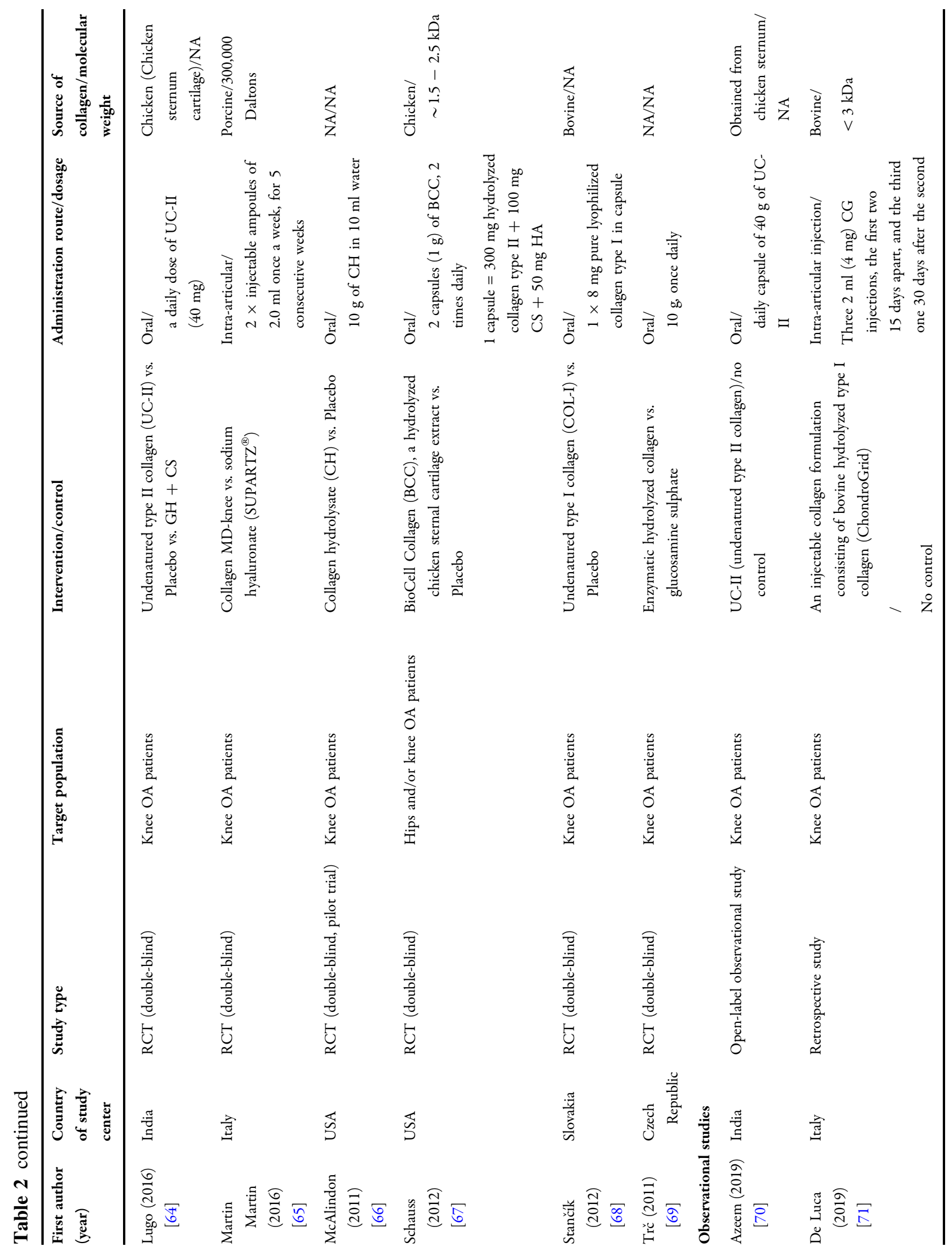




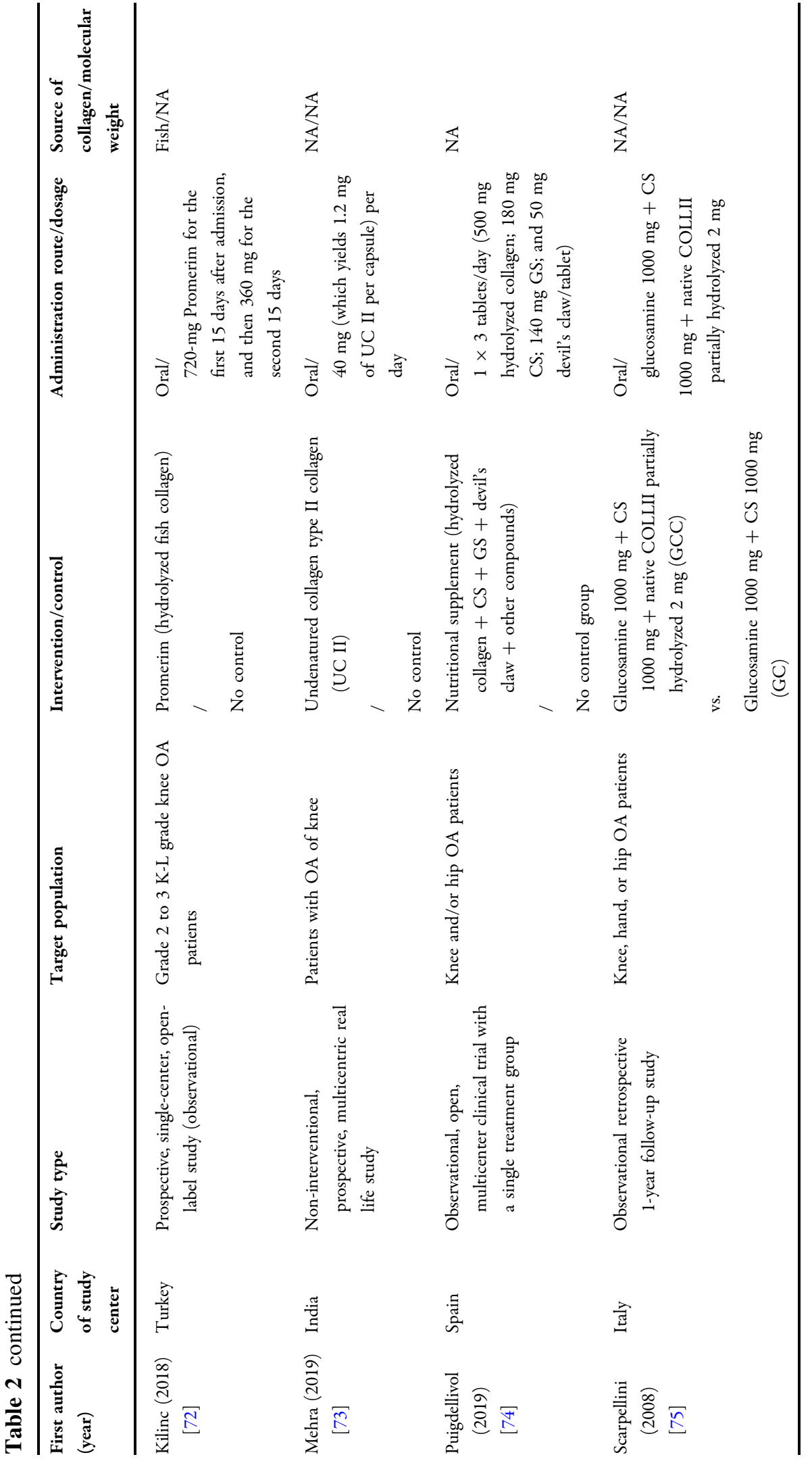




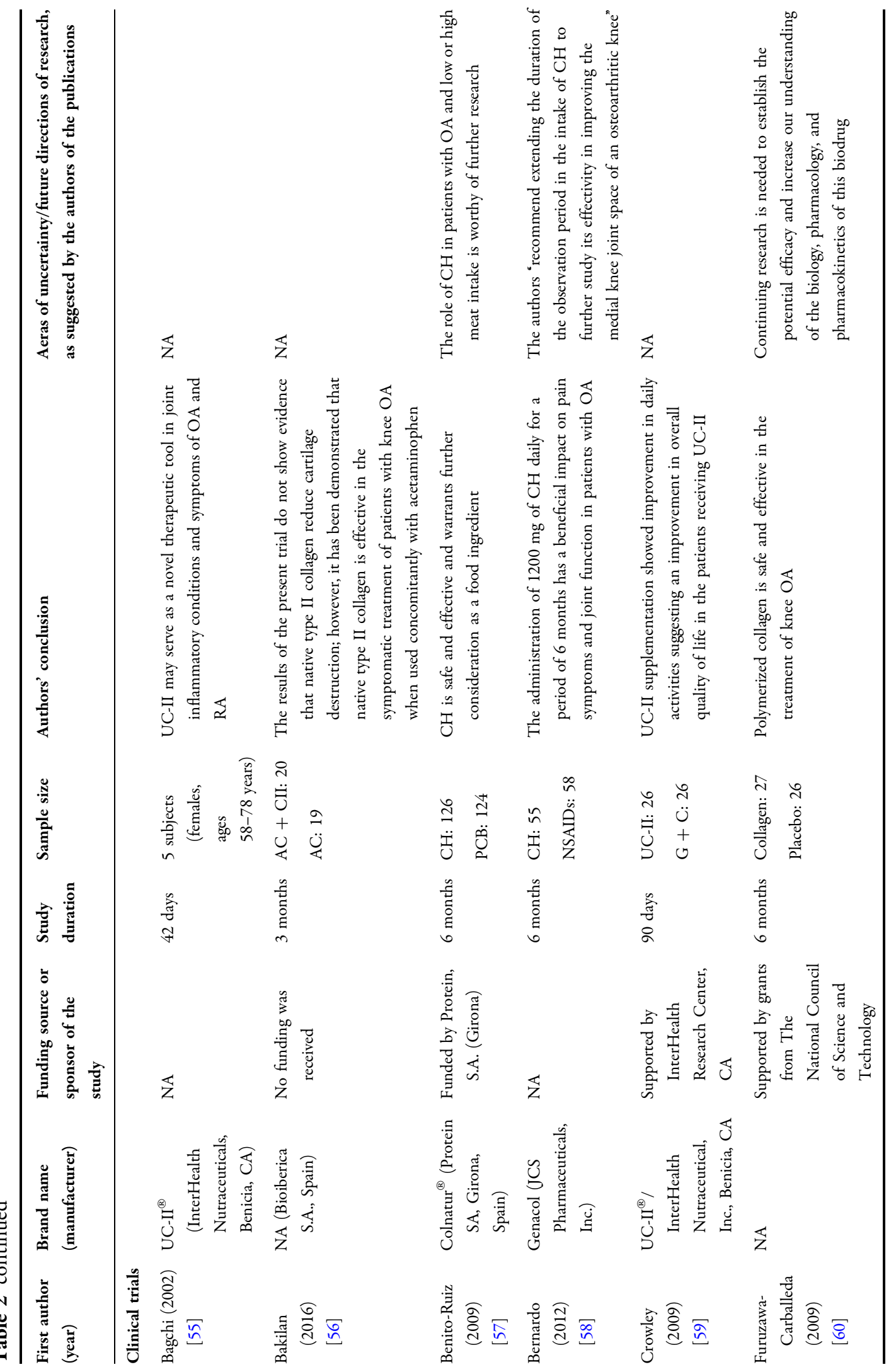




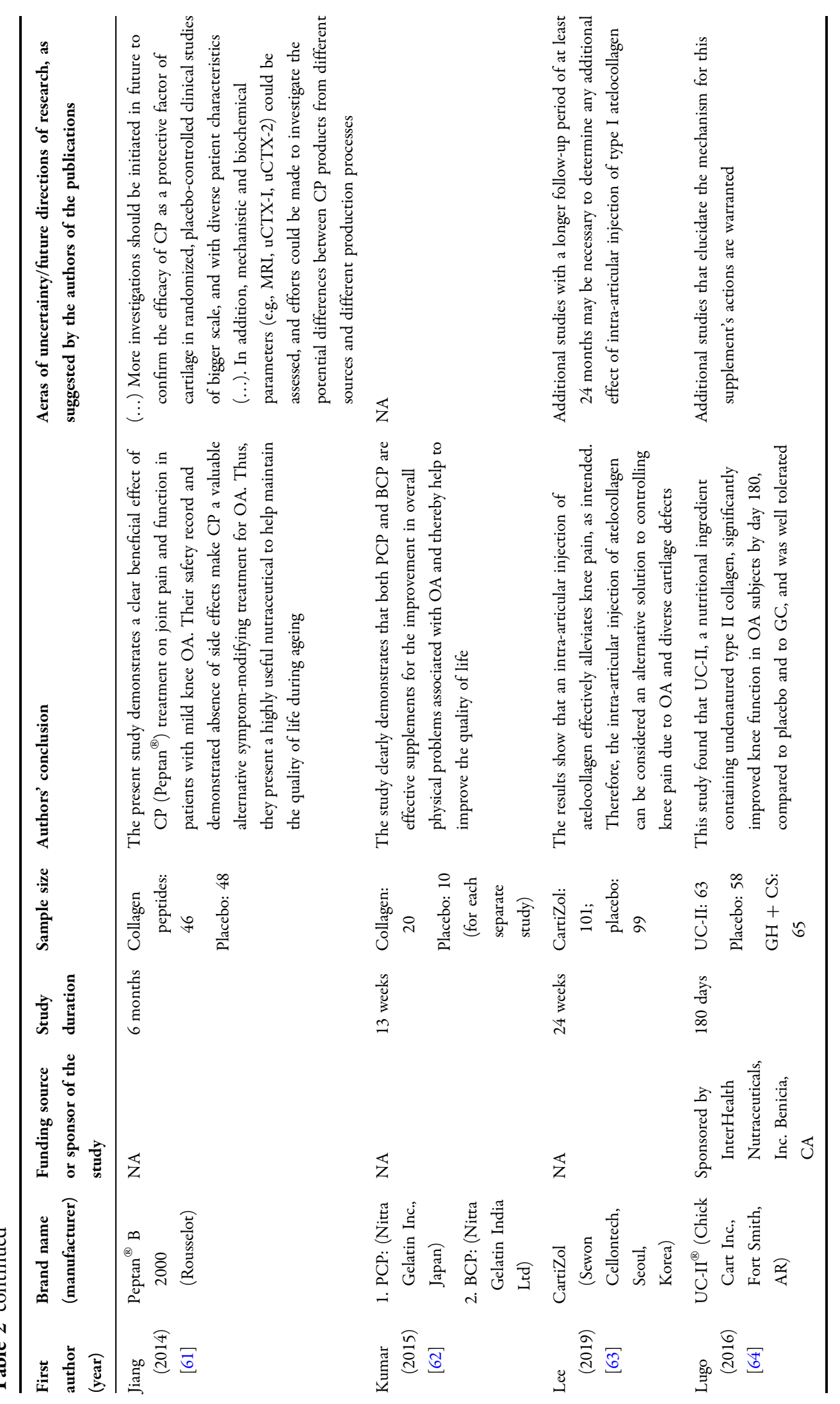




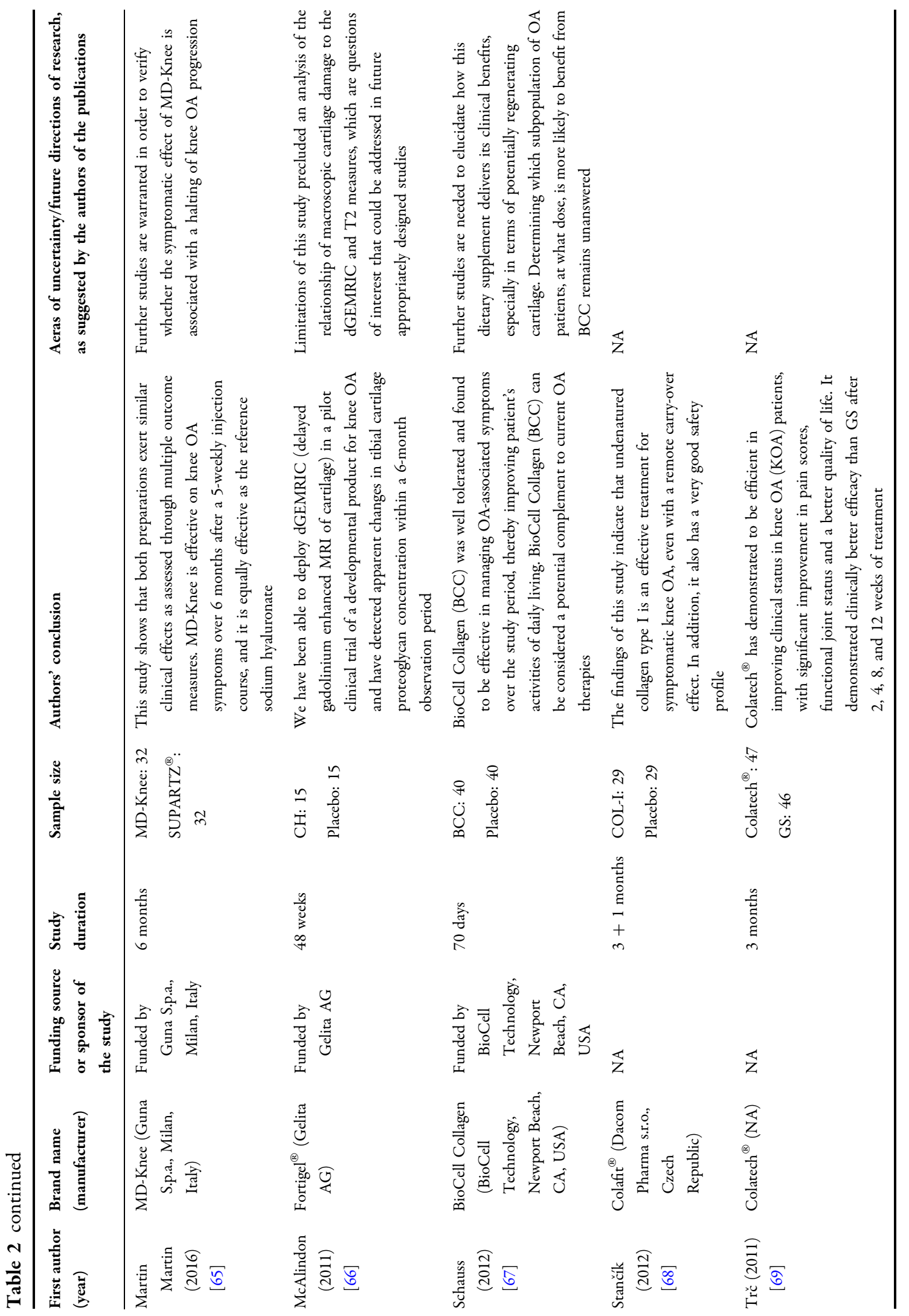




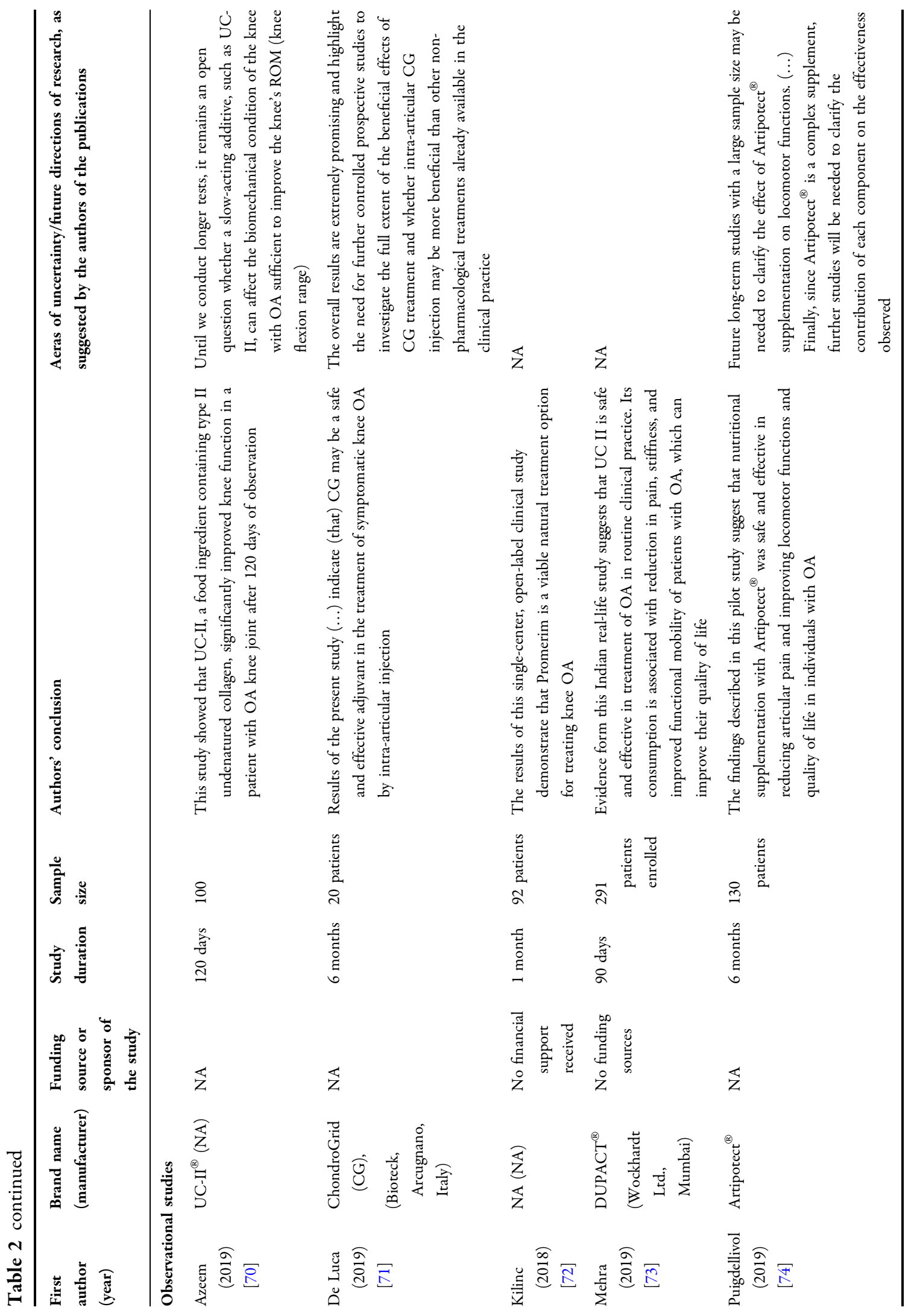




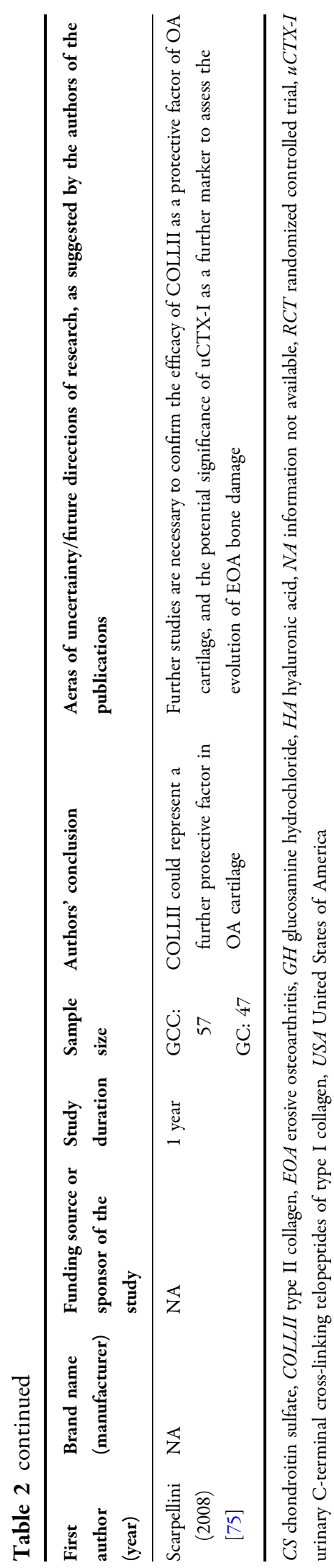

(UC-II). The other three studies on UC investigated all type II collagen: The study by Bagchi et al. [55] is a very small pilot study with no control group, associated to an in vitro mechanistic exploration study; according to the results from our literature search, this was the very first publication on collagen derivatives in $\mathrm{OA}$ and cartilage repair. In all of the five clinical trials on UC, orally administered UC was used (see Table 2).

We identified ten clinical trials on $\mathrm{CH}$, three of which used intra-articular $\mathrm{CH}$ and seven oral $\mathrm{CH}$. Among the studies on $\mathrm{CH}$, one investigated atelocollagen, which was intra-articularly injected [63]. Contrary to studies on UC, most clinical trials on $\mathrm{CH}$ in $\mathrm{OA}$ were placebo-controlled trials.

Most of the clinical trials on collagen derivatives (UC and $\mathrm{CH}$ ) were relatively shortterm studies (3-6 months), the longest study duration of currently available studies being 11 months (only one study). In addition, it is worth noting that almost all of these studies are of relatively small in size. Despite variations in dosages and sources of collagen, all of these studies on UC and $\mathrm{CH}$ concluded on positive effects in OA patients (see Table 2 for details).

Three major systematic reviews, all with meta-analysis, have evaluated the effects of collagen derivatives in OA patients [27, 28, 31]. These systematic reviews included different individual studies (Table 3), depending on their publication date and their selection criteria. The two latest systematic reviews/meta-analyses $[28,31]$ concluded on beneficial symptomatic effects of supplementation with collagen derivatives, but all advocated for longer clinical trials in larger populations to establish their therapeutic effects in OA patients.

\section{DISCUSSION}

The aim of this study was to systematically review the body of evidence on collagen derivatives in $\mathrm{OA}$ and cartilage repair, and describe the nature and trends of current research and research findings in this setting.

Our systematic literature search indicates that there are relatively few studies, both 


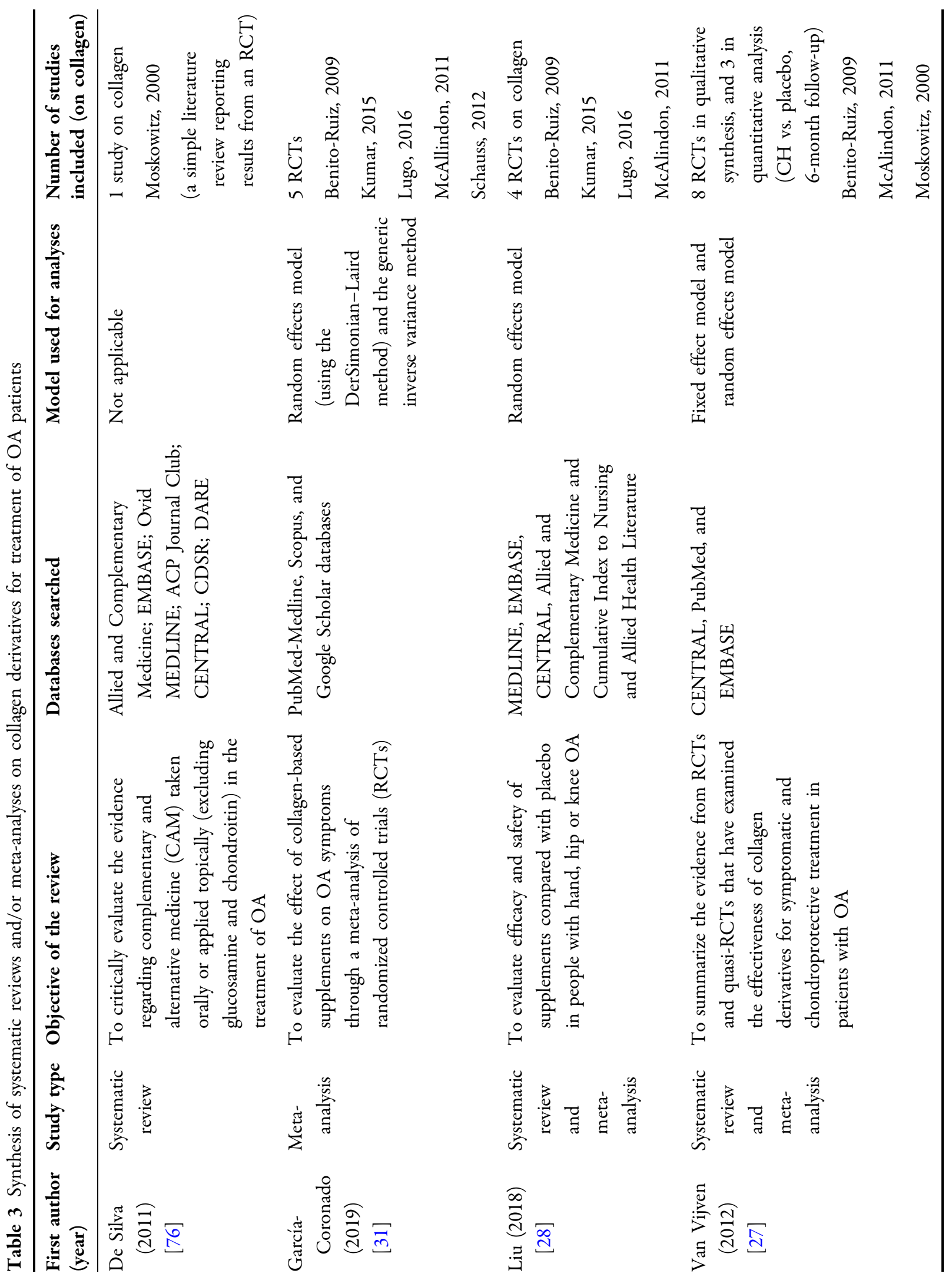




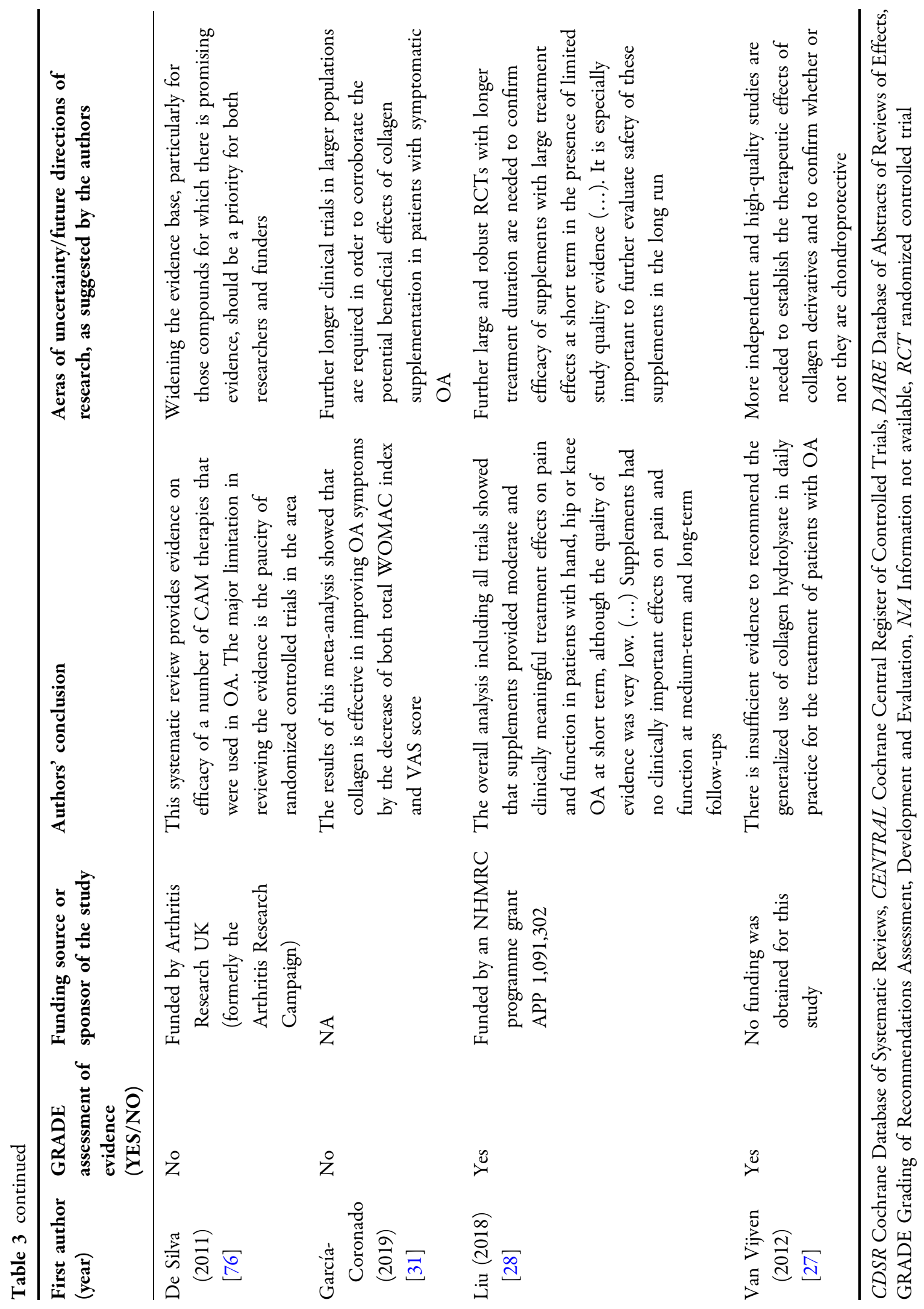


preclinical and clinical, on the effects of collagen derivatives in $\mathrm{OA}$ and cartilage repair. America and Asia are the parts of the world where most of the studies have been conducted, with only a quarter of studies published by research teams from European countries. Research interest in collagen derivatives as a supplement option for OA and cartilage repair seems to be relatively recent; in fact, the large part of the research in this setting has been undertaken in the past decade.

Our findings indicate that most of the research published so far, not taking into account its quality, has concluded on beneficial effects of collagen derivatives, suggesting that they may have some potential to serve as a novel and relevant supplement option for OA patients. In particular, all of the in vivo preclinical studies, whether on UC or $\mathrm{CH}$, and whatever the source (chicken, fish, porcine, bovine, squid) or molecular weight of collagen, have demonstrated a potential of collagen derivatives to reduce cartilage destruction or promote cartilage repair (see Table 1). These exciting results have, however, been moderated by findings from two in vitro studies conducted by the same research group from Germany, which compared different $\mathrm{CH}$ preparations (different sources and sizes) and concluded that $\mathrm{CHs}$ from various sources differed in terms of their peptide composition and were either ineffective or even detrimental to OA cartilage $[43,44]$. Similar findings were reported by a subsequent study from Thailand, published in 2018, which compared fish $\mathrm{CH}$ of different molecular weights [41]. Besides the results from these in vitro studies, the findings from the in vivo studies have been confirmed by clinical studies in OA patients. In fact, all of the published clinical trials found that oral supplementation with collagen derivatives or administration by intra-articular injection improved symptoms and/or quality of life in OA patients. However, it may be necessary to take these research conclusions with caution, as most of the clinical trials were of relatively short term and small in scale. In addition, there are currently very few RCTs against placebo on UC and $\mathrm{CH}$ in $\mathrm{OA}$ patients. Although most of the available clinical trials on $\mathrm{CH}$ (ten studies) are
RCTs against placebo, it should be kept in mind that these trials investigated $\mathrm{CHs}$ from various origins, molecular weights, and from different manufacturers (see Table 2). As reported by Schadow et al. [44], due to the high variability between $\mathrm{CH}$ preparations, no effect can be extrapolated from a $\mathrm{CH}$ to another one. Another important point is the quality of evidence from these studies, as reported by metaanalyses using the GRADE system. In fact, in a meta-analysis by Van Vijven et al. [27], the evidence was found to be of moderate to low quality, among others, because of methodological shortcomings in the included studies. Very importantly, our findings indicate that there is consensus among clinical research authors that further research is needed to confirm these preliminary results $[61,63,65]$ and, particularly, to elucidate the mechanisms of action $[61,64]$ of collagen derivatives and their effectivity in delaying the natural history of cartilage degradation in OA patients $[58,65,67]$. The need for high-quality and long-term clinical trials in larger populations has also been advocated by the authors of all the meta-analyses on collagen derivatives in OA $[27,28,31]$. On the other side, it is important to note that current evidence shows that collagen derivatives are safe for $\mathrm{OA}$ patients $[59,64]$, although further confirmatory studies are warranted [28]. However, the safety results seem to be confirmed by toxicity laboratory studies, both for UC [77] and $\mathrm{CH}$ [78].

Although better clinical trials and additional preclinical studies are warranted to confirm the beneficial effects of collagen derivatives in OA patients, many other studies in other populations have also shown promising results. In fact, several clinical trials in veterinary medicine have come to the conclusion that collagen derivatives may have beneficial effects in animals, with a good safety profile [79-81]. Likewise, studies on healthy subjects or those with articular pain have concluded on the efficacy of supplementation with collagen derivatives $[82,83]$, showing improvement of joint comfort in healthy subjects [84]. Additionally, a few studies have investigated the effect of collagen derivatives on chondrocytes in vitro, with promising results. These particular studies have not been included in the current scoping review 
(unless they also involved experiments on articular cartilage or OA) because they did not directly address the research question of this paper. For example, Oesser and Seifert showed that culture of bovine chondrocytes with degraded collagen stimulated the biosynthesis and secretion of type II collagen [85]. Likewise, a study included in this scoping review reported that $\mathrm{CH}$ may prompt chondrocytes to produce hyaline cartilage and prevent fibrous tissue formation [71].

One of the questions raised by the idea of supplementation with articular cartilage components such as collagen derivatives in $\mathrm{OA}$ patients is the gut absorption and the therapeutic mechanism behind the effects of such compounds. To answer the first part of that question, Oesser et al. [22] orally administered a ${ }^{14} \mathrm{C}$ labeled gelatine hydrolysate to mice in order to investigate the time course of its absorption and subsequent distribution in various tissues. They found that gelatine hydrolysate accumulated in cartilage tissue after intestinal absorption and distribution through blood circulation, a few hours after ingestion [22]. More recently, Taga et al. [24] reported concentration of collagen-specific peptides in human plasma and urine after oral ingestion. Similar results have been reported by other authors [23, 86, 87]. However, the real working mechanism of collagen derivatives in OA remains roughly unclear [44, 47], although preliminary results suggest the stimulation of collagen biosynthesis by chondrocytes [85], the use of collagen-specific peptides as building block for articular cartilage [22], or the inhibition of apoptosis and hypertrophy of chondrocytes [26]. Taken together, all of the results from the studies included in this scoping review, along with evidence from other studies, as briefly reported hereunder, strongly suggest that use of collagen derivatives as dietary supplements may be a relevant strategy in the therapeutic management of $\mathrm{OA}$, albeit this remains to be confirmed.

Another issue that needs to be clarified is the role of the source of collagen and manufacturing processes on its therapeutic effects. In fact, it has been reported that different $\mathrm{CH}$ may have different peptide composition $[43,44,88]$ and exhibit different effects on articular cartilage $[43,44]$. It may therefore be important to differentiate collagen derivatives according to source of collagen and manufacturing procedures. Setting up studies to identify therapeutically useful peptides in collagen derivatives may help in identifying the best sources of collagen. Research on the potential for difference in effects according to molecular weights also warrants further consideration, though current evidence already shows differences in effects on cartilage metabolism [41]. All of these parameters might, in fact, be relevant for future metaanalyses, particularly in the investigation of possible sources of inconsistent results from clinical trials.

While collating and synthetizing evidence from individual studies, we identified several gaps in the current research on collagen derivatives in OA. Firstly, regarding preclinical studies, there are very few in vitro studies assessing the effects of collagen derivatives on articular cartilage. Another important point is the lack of harmonization of endpoints or outcomes in preclinical studies; in fact, using the same endpoints for the same types of studies may ease comparability of results across studies and limit unnecessary controversies. The second type of research gap is related to clinical studies. As previously outlined, there is a lack of long-term and large-scale randomized placebocontrolled trials in humans to support the beneficial effects of collagen derivatives in OA, a gap that has been identified by other authors $[28,31]$. Even if it may be argued that power calculation has been performed for the current studies, a meta-epidemiological study investigating the effects of small study in meta-analyses of OA trials concluded that results of small trials of low methodological quality often distort results of meta-analyses in OA [89]. The authors of this meta-epidemiolocal study suggested the randomization of an average of 100 patients per treatment arm to achieve satisfactory requirements [89]. Adhering to this for future OA trials, whatever the outcomes considered, may help in improving consensus around the findings from clinical studies on collagen derivatives in OA. The other important research gap identified is the lack of studies 
involving patients with OA of joints other than the knee; indeed, having positive results from high-quality studies including patients with hip or hand OA may strengthen the body of evidence on the role of collagen derivatives in OA. Likewise, clinical studies investigating radiological changes in OA patients after supplementation with collagen derivatives are still lacking. Ultimately, future clinical research authors should consider adhering to the guidelines developed by the European Society for Clinical and Economic Aspects of Osteoporosis, Osteoarthritis and Musculoskeletal Diseases (ESCEO) for reporting of adverse events in manuscripts on clinical trials on collagen derivatives in OA patients, for transparency around the safety of these compounds [90].

This scoping review has some limitations that need to be outlined. One of the limitations is the fact that we did not report the outcomes considered in the individual studies. While reporting outcomes in the tables synthetizing the studies would have been highly relevant, particularly for preclinical studies, doing this appeared challenging for a reason of readability of information in the tables. We would therefore advise the reader of this research to go through the specific articles in the case he/she would need this specific information. Otherwise, the main outcomes in these studies are anyway somehow evoked in the conclusions reported in Tables 1 and 2. Another potential limitation is the fact that we did not include studies investigating the effects of collagen derivatives on chondrocytes, which would allow covering the whole research area of collagen derivatives in $\mathrm{OA}$ and cartilage repair. However, we thought it would be better to first focus on studies directly investigating the effects of collagen derivatives on articular cartilage or in OA patients, before envisaging looking at studies on chondrocytes, which may be more mechanism exploration studies. Not having assessed the methodological quality of the studies included in this review may also be considered another limitation; however, scoping reviews do not traditionally investigate this issue $[37,38]$.

\section{CONCLUSIONS}

This study is the very first systematic scoping review on collagen derivatives in OA. It provides a clear overview of the current knowledge, from preclinical to real-life studies, on the effect of collagen derivatives on articular cartilage and symptoms in OA patients. Current evidence shows that supplementation with collagen derivatives, as food ingredient (oral $\mathrm{CH}$ or UC) or biodrug (intra-articular $\mathrm{CH}$ ), may be a potential therapeutic or supportive strategy in OA patients, although much more work is needed in terms of laboratory and clinical research before any definitive conclusion can be made. Clarifying the mechanism of action of collagen derivatives in $\mathrm{OA}$, designing and conducting better and further randomized placebo-controlled trials with specific products manufactured according to Good Manufacturing Practice, and performing high-quality metaanalyses according to brands using data from high-quality studies, will help in bringing robust evidence on the usefulness or not of collagen derivatives as a relevant complementary secondary prevention option for the management of OA.

\section{ACKNOWLEDGEMENTS}

Funding. This research was partly supported by Lonza Consumer Health Ingredient Inc. However, the conception and writing of the protocol, the collection and synthesis of the data and their interpretation, the drafting of this manuscript, and the decision to submit it for publication, were the sole responsibility of the authors, without any influence of any third party. No Rapid Service Fee was received by the journal for the publication of this article.

Authorship. All named authors meet the International Committee of Medical Journal Editors (ICMJE) criteria for authorship for this article, take responsibility for the integrity of the work as a whole, and have given their approval for this version to be published. 
Disclosures. Germain Honvo has received lecture fees and travel support from IBSA, outside the submitted work. Olivier Bruyère has received grants from Biophytis, IBSA, MEDA, Servier, SMB, and Theramex, outside the submitted work. Olivier Bruyère is also a member of the journal's Editorial Board. Jean-Yves Reginster has received grants from IBSA-Genevrier, Mylan, CNIEL, and Radius Health (through their institution); consulting fees from IBSA Genevrier, Mylan, CNIEL, Radius Health, and Pierre Fabre; fees for participation in review activities from IBSA-Genevrier, MYLAN, CNIEL, Radius Health, and Teva; payment for lectures from AgNovos, CERIN, CNIEL, Dairy Research Council (DRC), Echolight, IBSA-Genevrier, Mylan, Pfizer Consumer Health, Teva, and Theramex, outside of the submitted work. Laetitia Lengelé and Alexia Charles have nothing to disclose.

Compliance with Ethics Guidelines. This article is based on previously conducted studies and does not contain any studies with human participants or animals performed by any of the authors.

Open Access. This article is licensed under a Creative Commons Attribution-NonCommercial 4.0 International License, which permits any non-commercial use, sharing, adaptation, distribution and reproduction in any medium or format, as long as you give appropriate credit to the original author(s) and the source, provide a link to the Creative Commons licence, and indicate if changes were made. The images or other third party material in this article are included in the article's Creative Commons licence, unless indicated otherwise in a credit line to the material. If material is not included in the article's Creative Commons licence and your intended use is not permitted by statutory regulation or exceeds the permitted use, you will need to obtain permission directly from the copyright holder. To view a copy of this licence, visit http://creativecommons.org/licenses/by$\mathrm{nc} / 4.0 /$.

\section{REFERENCES}

1. Vos T, Abajobir AA, Abbafati C, et al. Global, regional, and national incidence, prevalence, and years lived with disability for 328 diseases and injuries for 195 countries, 1990-2016: a systematic analysis for the Global Burden of Disease Study 2016. Lancet. 2017;390:1211-59.

2. Cross M, Smith E, Hoy D, et al. The global burden of hip and knee osteoarthritis: estimates from the global burden of disease 2010 study. Ann Rheum Dis. 2014;73:1323-30.

3. Bitton R. The economic burden of osteoarthritis. Am J Manag Care. 2009;15:S230-S235235.

4. Salmon JH, Rat AC, Sellam J, et al. Economic impact of lower-limb osteoarthritis worldwide: a systematic review of cost-of-illness studies. Osteoarthr Cartil. 2016;24:1500-8.

5. Neogi T. The epidemiology and impact of pain in osteoarthritis. Osteoarthr Cartil. 2013;21:1145-53.

6. Litwic A, Edwards MH, Dennison EM, Cooper C. Epidemiology and burden of osteoarthritis. Br Med Bull. 2013;105:185-99.

7. Berthelot G, Johnson S, Noirez P, et al. The ageperformance relationship in the general population and strategies to delay age-related decline in performance. Arch Public Heal. 2019;77:1-9.

8. Bruyère $\mathrm{O}$, Honvo $\mathrm{G}$, Veronese $\mathrm{N}$, et al. An updated algorithm recommendation for the management of knee osteoarthritis from the European Society for Clinical and Economic Aspects of Osteoporosis, Osteoarthritis and Musculoskeletal Diseases (ESCEO). Semin Arthritis Rheum. 2019;49:337-50.

9. Bannuru RR, Osani MC, Vaysbrot EE, et al. OARSI guidelines for the non-surgical management of knee, hip, and polyarticular osteoarthritis. Osteoarthr Cartil. 2019;27:1578-89.

10. Yusuf E. Pharmacologic and non-pharmacologic treatment of osteoarthritis. Curr Treat Options Rheumatol. 2016;2:111-25.

11. McAlindon TE, Bannuru RR, Sullivan MC, et al OARSI guidelines for the non-surgical management of knee osteoarthritis. Osteoarthr Cartil. 2014;22: 363-88.

12. Bruyère $\mathrm{O}$, Cooper $\mathrm{C}$, Pelletier $\mathrm{JP}$, et al. An algorithm recommendation for the management of knee osteoarthritis in Europe and internationally: a report from a task force of the European Society for Clinical and Economic Aspects of Osteoporosis and 
Osteoarthritis (ESCEO). Semin Arthritis Rheum. 2014;44:253-63.

13. Machado GC, Maher CG, Ferreira PH, et al. Efficacy and safety of paracetamol for spinal pain and osteoarthritis: systematic review and meta-analysis of randomised placebo-controlled trials. BMJ. 2015;350:h1225-h12251225.

14. Roberts E, Nunes VD, Buckner S, et al. Paracetamol: Not as safe as we thought? A systematic literature review of observational studies. Ann Rheum Dis. 2016;75:552-9.

15. Olsen AMS, Fosbøl EL, Lindhardsen J, et al. Longterm cardiovascular risk of nonsteroidal anti-inflammatory drug use according to time passed after first-time myocardial infarction: a nationwide cohort study. Circulation. 2012;126:1955-63.

16. Curtis E, Fuggle N, Shaw S, et al. Safety of cyclooxygenase- 2 inhibitors in osteoarthritis: outcomes of a systematic review and meta-analysis. Drugs Aging. 2019;36:25-44.

17. Bruyere O, Reginster J. Glucosamine and chondroitin sulfate as therapeutic agents for knee and hip osteoarthritis. Drugs Aging. 2007;24:573-80.

18. Reginster JY, Veronese N. Highly purified chondroitin sulfate: a literature review on clinical efficacy and pharmacoeconomic aspects in osteoarthritis treatment. Aging Clin Exp Res. 2020. https://doi.org/10.1007/s40520-020-01643-8.

19. Deal CL, Moskowitz RW. Nutraceuticals as therapeutic agents in osteoarthritis. The role of glucosamine, chondroitin sulfate, and collagen hydrolysate. Rheum Dis Clin N Am. 1999;25: 379-95.

20. Bello AE, Oesser S. Collagen hydrolysate for the treatment of osteoarthritis and other joint disorders: a review of the literature. Curr Med Res Opin. 2006;22:2221-32.

21. Apostu D, Lucaciu O, Mester A, et al. Systemic drugs with impact on osteoarthritis. Drug Metab Rev. 2019;51:498-523.

22. Oesser S, Adam M, Babel W, Seifert J. Oral administration of $14 \mathrm{C}$ labeled gelatin hydrolysate leads to an accumulation of radioactivity in cartilage of mice (C57/BL). J Nutr. 1999;129:1891-5.

23. Yamamoto S, Deguchi K, Onuma M, Numata N, Sakai Y. Absorption and urinary excretion of peptides after collagen tripeptide ingestion in humans. Biol Pharm Bull. 2016;39:428-34.

24. Taga Y, Iwasaki Y, Shigemura Y, Mizuno K. Improved in vivo tracking of orally administered collagen hydrolysate using stable isotope labeling and LC-MS techniques. J Agric Food Chem. 2019;67:4671-8.

25. Bagi CM, Berryman ER, Teo S, Lane NE. Oral administration of undenatured native chicken type II collagen (UC-II) diminished deterioration of articular cartilage in a rat model of osteoarthritis (OA). Osteoarthr Cartil. 2017;25:2080-90.

26. Dai M, Sui B, Xue Y, Liu X, Sun J. Cartilage repair in degenerative osteoarthritis mediated by squid type II collagen via immunomodulating activation of M2 macrophages, inhibiting apoptosis and hypertrophy of chondrocytes. Biomaterials. 2018;180: 91-103.

27. Van Vijven JPJ, Luijsterburg PAJ, Verhagen AP, van Osch GJVM, Kloppenburg M, Bierma-Zeinstra SMA. Symptomatic and chondroprotective treatment with collagen derivatives in osteoarthritis: a systematic review. Osteoarthr Cartil. 2012;20:809-21.

28. Liu X, Machado GC, Eyles JP, Ravi V, Hunter DJ. Dietary supplements for treating osteoarthritis: a systematic review and meta-analysis. Br J Sports Med. 2018;52:167-75.

29. Fuggle NR, Cooper C, Oreffo ROC, et al. Alternative and complementary therapies in osteoarthritis and cartilage repair. Aging Clin Exp Res. 2020;32: 547-60.

30. Honvo G, Reginster JY, Rabenda V, et al. Safety of symptomatic slow-acting drugs for osteoarthritis: outcomes of a systematic review and meta-analysis. Drugs Aging. 2019;36:65-99.

31. García-Coronado JM, Martínez-Olvera L, ElizondoOmaña RE, et al. Effect of collagen supplementation on osteoarthritis symptoms: a meta-analysis of randomized placebo-controlled trials. Int Orthop. 2019;43:531-8.

32. Kloppenburg M, Kroon FPB, Blanco FJ, et al. 2018 update of the EULAR recommendations for the management of hand osteoarthritis. Ann Rheum Dis. 2019;78:16-24.

33. Bruyère O, Cooper C, Al-Daghri NM, Dennison EM, Rizzoli R, Reginster JY. Inappropriate claims from non-equivalent medications in osteoarthritis: a position paper endorsed by the European Society for Clinical and Economic Aspects of Osteoporosis, Osteoarthritis and Musculoskeletal Diseases (ESCEO). Aging Clin Exp Res. 2018;30:111-7.

34. Honvo $G$, Bruyère $O$, Reginster JY. Update on the role of pharmaceutical-grade chondroitin sulfate in the symptomatic management of knee osteoarthritis. Aging Clin Exp Res. 2019;31:1163-7. 
35. Eriksen P, Bartels EM, Altman RD, Bliddal H, Juhl C, Christensen R. Risk of bias and brand explain the observed inconsistency in trials on glucosamine for symptomatic relief of osteoarthritis: a meta-analysis of placebo-controlled trials. Arthritis Care Res. 2014;66:1844-55.

36. Honvo $\mathrm{G}$, Bruyère $\mathrm{O}$, Geerinck $\mathrm{A}$, Veronese $\mathrm{N}$, Reginster JY. Efficacy of chondroitin sulfate in patients with knee osteoarthritis: a comprehensive meta-analysis exploring inconsistencies in randomized, placebo-controlled trials. Adv Ther. 2019;36:1085-99.

37. Peters MDJ, Godfrey CM, Khalil H, McInerney P, Parker D, Soares CB. Guidance for conducting systematic scoping reviews. Int J Evid Based Healthc. 2015;13:141-6.

38. Arksey H, O’Malley L. Scoping studies: towards a methodological framework. Int J Soc Res Methodol Theory Pract. 2005;8:19-32.

39. Levac D, Colquhoun H, O'Brien KK. Scoping studies: advancing the methodology. Implement Sci. 2010;5:1-9.

40. Tricco AC, Lillie E, Zarin W, et al. PRISMA extension for scoping reviews (PRISMA-ScR): checklist and explanation. Ann Intern Med. 2018;169:467-73.

41. Boonmaleerat K, Wanachewin O, Phitak T, Pothacharoen P, Kongtawelert P. Fish collagen hydrolysates modulate cartilage metabolism. Cell Biochem Biophys. 2018;76:279-92.

42. Furuzawa-Carballeda J, Muñoz-Chablé OA, BarriosPayán J, Hernández-Pando R. Effect of polymerizedtype I collagen in knee osteoarthritis. I. In vitro study. Eur J Clin Investig. 2009;39:591-7.

43. Schadow S, Siebert HC, Lochnit G, Kordelle J, Rickert M, Steinmeyer J. Collagen metabolism of human osteoarthritic articular cartilage as modulated by bovine collagen hydrolysates. PLoS ONE. 2013;8:e53955.

44. Schadow S, Simons VS, Lochnit G, et al. Metabolic response of human osteoarthritic cartilage to biochemically characterized collagen hydrolysates. Int J Mol Sci. 2017;18:207.

45. Dai M, Liu X, Wang N, Sun J. Squid type II collagen as a novel biomaterial: isolation, characterization, immunogenicity and relieving effect on degenerative osteoarthritis via inhibiting STAT1 signaling in pro-inflammatory macrophages. Mater Sci Eng C. 2018;89:283-94.

46. Dar QA, Schott EM, Catheline SE, et al. Daily oral consumption of hydrolyzed type 1 collagen is chondroprotective and antiinflammatory in murine posttraumatic osteoarthritis. PLOS ONE. 2017;12:1-24.

47. Di Cesare ML, Micheli L, Zanardelli M, Ghelardini C. Low dose native type II collagen prevents pain in a rat osteoarthritis model. BMC Musculoskelet Disord. 2013;14:228.

48. Hashida M, Miyatake K, Okamoto Y, et al. Synergistic effects of D-glucosamine and collagen peptides on healing experimental cartilage injury. Macromol Biosci. 2003;3:596-603.

49. Isaka S, Someya A, Nakamura S, et al. Evaluation of the effect of oral administration of collagen peptides on an experimental rat osteoarthritis model. Exp Ther Med. 2017;13:2699-706.

50. Nakatani S, Mano H, Sampei C, Shimizu J, Wada M. Chondroprotective effect of the bioactive peptide prolyl-hydroxyproline in mouse articular cartilage in vitro and in vivo. Osteoarthr Cartil. 2009;17: 1620-7.

51. Naraoka T, Ishibashi Y, Tsuda E, Yamamoto Y, Kusumi T, Toh S. Periodic knee injections of collagen tripeptide delay cartilage degeneration in rabbit experimental osteoarthritis. Arthritis Res Ther. 2013;15:1-10.

52. Ohara $\mathrm{H}$, Iida $\mathrm{H}$, Ito $\mathrm{K}$, Takeuchi $\mathrm{Y}$, Nomura $\mathrm{Y}$. Effects of Pro-Hyp, a collagen hydrolysate-derived peptide, on hyaluronic acid synthesis using in vitro cultured synovium cells and oral ingestion of collagen hydrolysates in a guinea pig model of osteoarthritis. Biosci Biotechnol Biochem. 2010;74: 2096-9.

53. Ohnishi A, Osaki T, Matahira Y, et al. Evaluation of the chondroprotective effects of glucosamine and fish collagen peptide on a rabbit ACLT model using serum biomarkers. J Vet Med Sci. 2013;75:421-9.

54. $\mathrm{Xu} \mathrm{D}$, Shen W. Chicken collagen type II reduces articular cartilage destruction in a model of osteoarthritis in rats. West Indian Med J. 2007;56: 202-7.

55. Bagchi D, Misner B, Bagchi M, Kothari SC, Downs BW, Preuss HG. Effects of orally administered undenatured type II collagen against arthritic inflammatory diseases: a mechanistic exploration. Int J Clin Pharmacol Res. 2002;22:101-10.

56. Bakilan F, Armagan O, Ozgen M, Tascioglu F, Bolluk $\mathrm{O}$, Alatas O. Effects of native type II collagen treatment on knee osteoarthritis: a randomized controlled trial. Eurasian J Med. 2016;48:95-101.

57. Benito-Ruiz P, Camacho-Zambrano MM, CarrilloArcentales JN, et al. A randomized controlled trial on the efficacy and safety of a food ingredient, 
collagen hydrolysate, for improving joint comfort. Int J Food Sci Nutr. 2009;60:99-113.

58. Bernardo MLR, Azarcon AC. A randomized controlled trial on the effects of oral collagen treatment on the medial knee joint space and functional outcome among patients diagnosed with osteoarthritis of the knee. PARM Proc. 2012;4:1-8.

59. Crowley DC, Lau FC, Sharma P, et al. Safety and efficacy of undenatured type II collagen in the treatment of osteoarthritis of the knee: a clinical trial. Int J Med Sci. 2009;6:312-21.

60. Furuzawa-Carballeda J, Muñoz-Chablé OA, MacíasHernández SI, Agualimpia-Janning A. Effect of polymerized-type i collagen in knee osteoarthritis. II. In vivo study. Eur J Clin Investig. 2009;39: 598-606.

61. Jiang J-X, Zhou J-L, Prawitt J. Collagen peptides improve knee osteoarthritis in elderly women: a 6-month randomized, double-blind, placebo-controlled study. Agro FOOD Industry $\mathrm{Hi}$ Tech. 2014;25:19-23.

62. Kumar S, Sugihara F, Suzuki K, Inoue N, Venkateswarathirukumara S. A double-blind, placebo-controlled, randomised, clinical study on the effectiveness of collagen peptide on osteoarthritis. J Sci Food Agric. 2015;95:702-7.

63. Lee HS, Oh KJ, Moon YW, In Y, Lee HJ, Kwon SY. Intra-articular injection of type I atelocollagen to alleviate knee pain: a double-blind, randomized controlled trial. Cartilage. 2019. https://doi.org/10. $1177 / 1947603519865304$.

64. Lugo JP, Saiyed ZM, Lane NE. Efficacy and tolerability of an undenatured type II collagen supplement in modulating knee osteoarthritis symptoms: a multicenter randomized, double-blind, placebocontrolled study. Nutr J. 2016;15:14.

65. Martin Martin LS, Massafra U, Bizzi E, Migliore A. A double blind randomized active-controlled clinical trial on the intra-articular use of Md-Knee versus sodium hyaluronate in patients with knee osteoarthritis ("Joint"). BMC Musculoskelet Disord. $2016 ; 17: 1-8$.

66. McAlindon TE, Nuite M, Krishnan N, et al. Change in knee osteoarthritis cartilage detected by delayed gadolinium enhanced magnetic resonance imaging following treatment with collagen hydrolysate: a pilot randomized controlled trial. Osteoarthr Cartil. 2011;19:399-405.

67. Schauss AG, Stenehjem J, Park J, Endres JR, Clewell A. Effect of the novel low molecular weight hydrolyzed chicken sternal cartilage extract, biocell collagen, on improving osteoarthritis-related symptoms: a randomized, double-blind, placebocontrolled trial. J Agric Food Chem. 2012;60: 4096-101.

68. Stančík R, Zvarka J, Hlaváč M, Kubinec V, Rovenský J. Collagen type I in the treatment of painful osteoarthritis of the knee. Reumatologia. 2012;50: 390-6.

69. Trč T, Bohmová J. Efficacy and tolerance of enzymatic hydrolysed collagen (EHC) vs. glucosamine sulphate (GS) in the treatment of knee osteoarthritis (KOA). Int Orthop. 2011;35:341-8.

70. Azeem MA, Patil R. The study of undenatured type II collagen in the knee osteoarthritis. Int J Orthop Traumatol Surg Sci. 2019;5:172-5.

71. De Luca P, Colombini A, Carimati G, Beggio M, de Girolamo L, Volpi P. Intra-articular injection of hydrolyzed collagen to treat symptoms of knee osteoarthritis. A functional in vitro investigation and a pilot retrospective clinical study. J Clin Med. 2019;8:975.

72. Kilinc BE, Oc Y, Alibakan G, Bilgin E, Kanar M, Eren OT. An Observational 1-month trial on the efficacy and safety of Promerim for improving knee joint. Clin Med Insights Arthritis Musculoskelet Disord. 2018;11:1-4.

73. Mehra A, Anand $\mathrm{P}$, Borate $\mathrm{M}$, et al. A non-interventional, prospective, multicentric real life Indian study to assess safety and effectiveness of un-denatured type 2 collagen in management of osteoarthritis. Int J Res Orthop. 2019;5:315-20.

74. Puigdellivol J, Comellas Berenger C, Pérez Fernández MÁ, et al. Effectiveness of a dietary supplement containing hydrolyzed collagen, chondroitin sulfate, and glucosamine in pain reduction and functional capacity in osteoarthritis patients. J Diet Suppl. 2019;16:379-89.

75. Scarpellini M, Lurati A, Vignati G, et al. Biomarkers, type II collagen, glucosamine and chondroitin sulfate in osteoarthritis follow-up: the "Magenta osteoarthritis study". J Orthop Traumatol. 2008;9: $81-7$.

76. de Silva V, El-Metwally A, Ernst E, Lewith G, Macfarlane GJ. Evidence for the efficacy of complementary and alternative medicines in the management of osteoarthritis: a systematic review. Rheumatology. 2011;50:911-20.

77. Yoshinari O, Marone PA, Moriyama H, Bagchi M, Shiojima Y. Safety and toxicological evaluation of a novel, water-soluble undenatured type II collagen. Toxicol Mech Methods. 2013;23:491-9. 
78. Schauss AG, Merkel DJ, Glaza SM, Sorenson SR. Acute and subchronic oral toxicity studies in rats of a hydrolyzed chicken sternal cartilage preparation. Food Chem Toxicol. 2007;45:315-21.

79. Beynen AC, van Geene HW, Grim HV, Jacobs P, van der Vlerk T. Oral administration of gelatin hydrolysate reduces clinical signs of canine osteoarthritis in a double-blind, placebo-controlled trial. Am J Anim Vet Sci. 2010;5:95-9.

80. Gupta RC, Canerdy TD, Lindley J, et al. Comparative therapeutic efficacy and safety of type-II collagen (UC-II), glucosamine and chondroitin in arthritic dogs: Pain evaluation by ground force plate. J Anim Physiol Anim Nutr (Berl). 2012;96: 770-7.

81. Stabile M, Samarelli R, Trerotoli P, et al. Evaluation of the effects of undenatured type II collagen (UCII) as compared to robenacoxib on the mobility impairment induced by osteoarthritis in dogs. Vet Sci. 2019;6:72.

82. Bruyère $\mathrm{O}$, Zegels $\mathrm{B}$, Leonori $\mathrm{L}$, et al. Effect of collagen hydrolysate in articular pain: a 6-month randomized, double-blind, placebo-controlled study. Complement Ther Med. 2012;20:124-30.

83. Clark KL, Sebastianelli W, Flechsenhar KR, et al. 24-Week study on the use of collagen hydrolysate as a dietary supplement in athletes with activityrelated joint pain. Curr Med Res Opin. 2008;24: 1485-96.

84. Lugo JP, Saiyed ZM, Lau FC, et al. Undenatured type II collagen (UC-II ${ }^{\circledR}$ ) for joint support: a randomized, double-blind, placebo-controlled study in healthy volunteers. J Int Soc Sports Nutr. 2013;10:48.

85. Oesser S, Seifert J. Stimulation of type II collagen biosynthesis and secretion in bovine chondrocytes cultured with degraded collagen. Cell Tissue Res. 2003;311:393-9.

86. Ohara H, Matsumoto $\mathrm{H}$, Ito $\mathrm{K}$, Iwai $\mathrm{K}$, Sato $\mathrm{K}$. Comparison of quantity and structures of hydroxyproline-containing peptides in human blood after oral ingestion of gelatin hydrolysates from different sources. J Agric Food Chem. 2007;55:1532-5.

87. Walrand S, Chiotelli E, Noirt F, Mwewa S, Lassel T. Consumption of a functional fermented milk containing collagen hydrolysate improves the concentration of collagen-specific amino acids in plasma. J Agric Food Chem. 2008;56:7790-5.

88. Simons VS, Lochnit G, Wilhelm J, Ishaque B, Rickert M, Steinmeyer J. Comparative analysis of peptide composition and bioactivity of different collagen hydrolysate batches on human osteoarthritic synoviocytes. Sci Rep. 2018;8:1-10.

89. Nüesch E, Trelle S, Reichenbach S, et al. Small study effects in meta-analyses of osteoarthritis trials: meta-epidemiological study. BMJ. 2010;341:c3515.

90. Honvo $G$, Bannuru RR, Bruyère $O$, et al. Recommendations for the reporting of harms in manuscripts on clinical trials assessing osteoarthritis drugs: a consensus statement from the European Society for clinical and economic aspects of osteoporosis, osteoarthritis and musculoskeletal diseases. Drugs Aging. 2019;36:145-59. 